\title{
Gravitational redshifts in main-sequence and giant stars ${ }^{\star} \star \star \star$
}

\author{
L. Pasquini ${ }^{1}$, C. Melo ${ }^{1}$, C. Chavero ${ }^{2}$, D. Dravins ${ }^{3}$, H.-G. Ludwig ${ }^{4,6}$, P. Bonifacio ${ }^{4,5}$, and R. De La Reza ${ }^{2}$ \\ ${ }^{1}$ ESO - European Southern Observatory, Karl-Schwarzschild-Strasse 2, 85748 Garching bei München, Germany \\ e-mail: 1pasquin@eso.org \\ 2 Observatório Nacional, Rua General José Cristino 77, 20921-400 Rio de Janeiro, RJ, Brazil \\ 3 Lund Observatory, Box 43, 22100 Lund, Sweden \\ 4 GEPI, Observatoire de Paris, CNRS, Université Paris Diderot, Place Jules Janssen, 92190 Meudon, France \\ 5 Istituto Nazionale di Astrofisica, Osservatorio Astronomico di Trieste, via Tiepolo 11, 34143 Trieste, Italy \\ ${ }^{6}$ ZAH - Landessternwarte, Königstuhl 12, 69117 Heidelberg, Germany
}

Received 5 July 2010 / Accepted 5 November 2010

\section{ABSTRACT}

\begin{abstract}
Context. Precise analyses of stellar radial velocities is able to reveal intrinsic causes of the wavelength shifts of spectral lines (other than Doppler shifts due to radial motion), such as gravitational redshifts and convective blueshifts.

Aims. Gravitational redshifts in solar-type main-sequence stars are expected to be some $500 \mathrm{~m} \mathrm{~s}^{-1}$ greater than those in giants. We search for this difference in redshifts among groups of open-cluster stars that share the same average space motion and thus have the same average Doppler shift.

Methods. We observed 144 main-sequence stars and cool giants in the M67 open cluster using the ESO FEROS spectrograph and obtained radial velocities by means of cross-correlation with a spectral template. Binaries and doubtful members were not analyzed, and average spectra were created for different classes of stars.

Results. The M 67 dwarf and giant radial-velocity distributions are each well represented by Gaussian functions, which share the same apparent average radial velocity to within $\simeq 100 \mathrm{~m} \mathrm{~s}^{-1}$. In addition, dwarfs in M 67 appear to be dynamically hotter $\left.(\sigma=0.90 \mathrm{~km} \mathrm{~s})^{-1}\right)$ than giants $\left(\sigma=0.68 \mathrm{~km} \mathrm{~s}^{-1}\right)$.

Conclusions. We fail to detect any difference in the gravitational redshifts of giants and MS stars. This is probably because of the differential wavelength shifts produced by the different hydrodynamics of dwarf and giant atmospheres. Radial-velocity differences measured between unblended lines in averaged spectra vary with line-strength: stronger lines are more blueshifted in dwarfs than in giants, apparently removing any effect of the gravitational redshift. Synthetic high-resolution spectra are computed from three dimensional (3D) hydrodynamic model atmospheres for both giants and dwarfs, and synthetic wavelength shifts obtained. In agreement with observations, 3D models predict substantially smaller wavelength-shift differences than expected from gravitational redshifts only. The procedures developed could be used to test 3D models for different classes of stars, but will ultimately require high-fidelity spectra for measurements of wavelength shifts in individual spectral lines.
\end{abstract}

Key words. stars: fundamental parameters - galaxies: star clusters: individual: M 67 - stars: late-type - stars: atmospheres techniques: radial velocities - gravitation

\section{Introduction}

\subsection{Gravitational redshift}

Spectroscopic measurements of stellar radial velocities have reached levels of precision that not only allow us to perform new types of studies, but also require a clearer understanding of effects other than a star's barycentric motion that may cause displacements of spectral-line wavelengths. One such effect is the gravitational redshift originating from the propagation of light between different gravitational potentials at the source and the observer.

Any theory respecting conservation of energy requires that radiation leaving a gravitational field loses energy, and with the 20th century understanding of the photon and the relation

* Based on observations collected at ESO, La Silla, Chile, during the agreement between the Observatorio Nacional at Rio de Janeiro and ESO.

$\star \star$ Table 1 is available in electronic form at

http://www.aanda.org and also at the CDS via anonymous ftp to cdsarc.u-strasbg.fr (130.79.128.5) or via

http://cdsarc.u-strasbg.fr/viz-bin/qcat?]/A+A/526/A127 between its energy and wavelength, early predictions could be made about the wavelength displacement expected for radiation leaving various stars, and these effects have been sought observationally.

While a determination of an absolute wavelength shift requires the true stellar center-of-mass motion (and its induced Doppler shift) to be known, relative shifts can be determined between stars sharing a common space motion. This applies to the orbit-averaged velocity of binary stars (where each component shares the same systemic velocity) and stars in [open] clusters that share the same space velocity vector.

The first claimed confirmation of the predicted gravitational redshift came from the measurement of the apparent radial velocity of Sirius B, the white-dwarf companion to Sirius (Adams 1925), identified as the difference from the motion expected in its 50-year orbit. However, this faint white dwarf is overwhelmed by scattered light from the much brighter primary and the validity (and even the honesty!) of these early measurements has been questioned (Hetherington 1980; Greenstein et al. 1985).

Gravitational redshifts have since been more reliably determined for numerous white dwarfs in binary systems and in 
nearby open clusters such as the Hyades and the Pleiades, finding values typically on the order of $30-40 \mathrm{~km} \mathrm{~s}^{-1}$, as measured against wavelength positions in the spectra of ordinary stars. Far larger gravitational redshifts have also been identified from spectra of X-ray bursts on neutron-star surfaces.

The gravitational redshift is proportional to the gravitational potential at the stellar surface, so it is proportional to the stellar mass, but inversely to its radius $R$, such that $v_{\text {grav }}=G M / R c$ where $G$ is the gravitational constant. The expected solar gravitational redshift for light escaping from the solar photospheric surface to infinity is $636.486 \pm 0.024 \mathrm{~m} \mathrm{~s}^{-1}$ (Lindegren \& Dravins 2003) and - since the shift diminishes with distance from the stellar center as $r^{-1}$ - becomes $633.5 \mathrm{~m} \mathrm{~s}^{-1}$ for light intercepted at the Earth's mean distance from the Sun $\left(r=215 R_{\odot}\right)$. A solar spectral line instead formed at chromospheric heights $(30 \mathrm{Mm}$, say; $r=1.04 R_{\odot}$ ) will have its redshift decreased by some $20 \mathrm{~m} \mathrm{~s}^{-1}$, and a coronal line by perhaps $100 \mathrm{~m} \mathrm{~s}^{-1}$.

For other stars, the shift scales as $\left(M / M_{\odot}\right)\left(R / R_{\odot}\right)^{-1}$, or as $\left(g / g_{\odot}\right)\left(R / R_{\odot}\right)$, where $g$ is the surface gravity. For distant stars, additional lineshifts may originate from large-scale gravitational fields in the Milky Way galaxy. For instance, the spectra of stars in the central Galactic bulge $\left(R_{*} \simeq 1 \mathrm{kpc}\right)$ may be gravitationally redshifted by $300-400 \mathrm{~m} \mathrm{~s}^{-1}$, while stars in the Magellanic Clouds $\left(R_{*} \simeq 55 \mathrm{kpc}\right.$ ) might be blueshifted by a similar amount, as seen by an observer near the solar Galactocentric position at $R_{\text {obs }} \simeq 8.5 \mathrm{kpc}$ (Lindegren \& Dravins 2003).

Across the Hertzsprung-Russell diagram, the gravitational redshifts are not expected to vary much along the main sequence between $\mathrm{A} 5 \mathrm{~V}$ and $\mathrm{K} 0 \mathrm{~V}$ (being around $650 \mathrm{~m} \mathrm{~s}^{-1}$ ), but to reach twice that value for more massive early-B stars with $M \simeq 10 M_{\odot}$. For giants the shifts decrease substantially, and for red supergiants it should be only some 20 or $30 \mathrm{~m} \mathrm{~s}^{-1}$, three orders of magnitude smaller than for white dwarfs (Griffin 1982; Dravins 2005). Thus, the difference between the shifts in dwarf and giant stars is expected to be some $0.5 \mathrm{~km} \mathrm{~s}^{-1}$, orders of magnitude greater than current measurement precision. These values are significant, both to study the precise dynamics in binaryand multiple-star systems (Griffin 1982; Pourbaix et al. 2002), and distinguish the wavelength shifts caused by the stellar atmospheric dynamics of convective motions (e.g., Dravins 1982; Asplund 2005; Dravins et al. 2005). Together with photospheric line asymmetries, these intrinsic wavelength shifts represent significant diagnostic tools for probing the three dimensional (3D) structure of stellar atmospheres (Dravins 2008; Nagendra et al. 2009), a field where additional diagnostics are needed to reliablydifferentiate between the hydrodynamic models predicting significantly different chemical compositions, oscillation properties, and so on.

\subsection{Searching for shifts in ordinary stars}

At first sight, the Sun would appear to be a promising source for determining absolute gravitational redshifts, since the radial Sun-Earth motion is both very small and precisely measured. However, already long ago, spectral lines in the Sun were found to be shifted by different but comparable amounts, i.e., a variation was found in absorption-line wavelengths across the solar disk from its center towards the limb that was initially a mystery. This is now well understood as convective lineshifts, originating from a summation of contributions from hot and rising (thus locally blueshifted) elements, and cool and sinking ones, and various effects of observational perspective across the disk. Many efforts have been made to measure the solar gravitational redshift (and several publications claimed its detection), although, in hindsight, almost all of these studies were constrained by the limited understanding of the interplay with other mechanisms causing subtle wavelength shifts. In addition to the effects of atmospheric motions, the limited accuracy of laboratory wavelengths, and even the effects of pressure shifts come into play. The most convincing demonstration of a gravitational redshift in the solar spectrum was possibly that of Beckers (1977). The magnetic fields in the cores of sunspots inhibit convective motions and greatly reduce the amount of both convective wavelength shifts, and their center-to-limb variation, enabling rather precise wavelength shift measurements in magnetically insensitive lines. From these lines, Beckers deduced a shift of $613 \mathrm{~m} \mathrm{~s}^{-1}$, which is consistent with the expected magnitude.

As for white dwarfs, systematic wavelength displacements between ordinary stars may be identified in binaries sharing a common systemic velocity, and for stars in open clusters, moving in space with a sufficiently small velocity dispersion. The earliest of these attempts seems to have been by Finlay-Freundlich who, starting around 1914, tried to find statistical evidence of gravitational redshifts (for a lucid review, see Hentschel 1994). Somewhat later, Trumpler (1935) recognized that massive $\mathrm{O}$ stars should be excellent targets, because they have large gravitational redshift and, at the same time they appear in young clusters where they, being massive, by equipartition of energy should have the smallest velocity dispersion. In some clusters, shifts of some $10 \mathrm{~km} \mathrm{~s}^{-1}$ were found relative to comparison stars, which were indicative of enormous stellar masses. A later understanding of these stars showed that these shifts should be much smaller (Conti et al. 1977), and as the effects of stellar variability, line asymmetries, and differences between the apparent velocities of different spectral features became appreciated, a certain amount of literature grew on suggested "anomalous" redshifts in binary high-luminosity stars, and other objects.

Only after the measurement precisions for individual stars had reached levels of around $100 \mathrm{~m} \mathrm{~s}^{-1}$, did it become practical to search for systematic differences in apparent velocities among ordinary stars, e.g., between main-sequence dwarfs and giants. One hint came from a study of the open cluster NGC 3680 by Nordström et al. (1997), who found the giants (though the sample contained only six of these stars) to be blueshifted on average by $0.4 \mathrm{~km} \mathrm{~s}^{-1}$ relative to the dwarfs, as expected for gravity.

For certain groups of stars, including those in undispersed moving clusters, absolute stellar radial motion can be determined from precise astrometry, without invoking any spectroscopy. The difference in spectroscopic lineshifts then reveals shifts intrinsic to stellar atmospheres. In the Hyades (Madsen et al. 2002), these signatures were seen for some classes of hotter main-sequence stars, but the spectral displacements of the (only three) Hyades K-giants did not show any significant deviation from that of main-sequence K-type stars.

Differences between the gravitational redshifts of dwarf and giant stars have thus been sought for almost a century. The value and usefulness of these measurements have been pointed out from time to time (e.g., von Hippel 1996) but conclusive or statistically significant observations have been lacking, a lack we aim to reduce with our present project.

\section{Radial velocities in open clusters}

Our aim is to observe the apparent radial velocities of mainsequence and giant stars in some stellar cluster(s) with sufficient precision to identify expected differences between the gravitational redshifts of these groups. The ideal cluster should 
obviously be populous and have stars covering a large range of $M / R$ ratios.

Not all clusters are suitable for this study. To ensure a good measurement precision for spectroscopic radial velocity, the stars must have spectral lines that are both narrow and numerous, which excludes young clusters where main-sequence stars are still rapidly rotating, producing broadened lines. In addition, higher-temperature giants are unsuitable because they display few spectral lines and often complex atmospheric dynamics. Thus, the cluster must be sufficiently old, with main-sequence rotation slower than some $5-10 \mathrm{~km} \mathrm{~s}^{-1}$, and must have a well developed red-giant branch with many cool giants that are good targets for radial-velocity measurements (e.g., Setiawan et al. 2004).

The relatively old cluster M 67 (NGC 2682) at a distance of $\sim 850 \mathrm{pc}$ was chosen as a nearly ideal object. Besides having a well-developed red-giant branch, it is a good target because its composition is almost solar (Tautvaišienè et al. 2000; Randich et al. 2006; Pace et al. 2008) and the age somewhat comparable, at 2.6 Gy (WEBDA 2009). It has been well studied in various aspects (Pasquini et al. 2008; Sandquist 2004), including as part of a census of binaries (Latham et al. 1992). Finally, we had already observed a homogeneous group of stars in M 67 (Melo et al. 2001; hereafter Paper I), verifying the modest rotational velocities, and could use these data as a starting point.

From previous radial-velocity data (e.g., Mathieu et al. 1986), the internal velocity dispersion of the space motions within M 67 was found to be $\approx 0.5-0.8 \mathrm{~km} \mathrm{~s}^{-1}$, similar to that deduced from proper motions (Girard et al. 1989). Other studies suggest that expansion and rotation of the cluster core are smaller than $1 \mathrm{~km} \mathrm{~s}^{-1}$ (Zhao et al. 1996; Loktin 2005).

Since these numbers are comparable to the expected difference in gravitational redshifts between dwarfs and giants, averages over many stars will be required, and attention must be paid to calibrating radial-velocity measurements between stars of different spectral types, identifying stellar multiplicity, possibly different velocities for stars mass-segregated into different parts of the cluster, and the physics of line formation in dynamic stellar atmospheres. In any case, since the internal measurement precision of modern radial-velocity instruments is very much better than this dispersion, significant progress should be possible.

\section{Observations and data reduction}

An observing program was carried out with FEROS (Fiberfed Extended Range Optical Spectrograph; Kaufer et al. 1999) in three different runs. The first took place during FEROS commissioning in October and November 1998, observing 28 mainsequence, turnoff, and giant stars. The radial velocities and $V \sin i$ were published in Paper I and are listed in the online Appendix for sake of completeness. Additionally, 68, mostly main-sequence and turnoff stars, were observed over four nights during the first week of February 2002 (Run II) in parallel with other projects during Brazilian time. Finally in Run III, 66 additional M 67 members were observed in late February 2008. For the first two runs, the spectrograph was attached to the ESO $1.5-\mathrm{m}$ telescope, and during the third to the MPG/ESO 2.2-m telescope. In total, 162 observations for 144 different stars were obtained.

FEROS is a bench-mounted, thermally controlled, prismcross-dispersed échelle spectrograph. It offers rather high resolution $(R \simeq 48000)$, and in a single recording with 39 échelle orders provides almost complete spectral coverage across the range $\approx 350-920 \mathrm{~nm}$. For bright stars, FEROS has been demonstrated to be precise to better than $20 \mathrm{~m} \mathrm{~s}^{-1}$ (Setiawan et al. 2004). Our targets are substantially fainter, but an exceedingly high precision is not required. The M 67 velocity dispersion is on the order of $1 \mathrm{~km} \mathrm{~s}^{-1}$, so a precision of a few hundred meters in a single exposure is sufficient. Exposure times were set to have a $S / N$ of around 30 or higher. With this level of photon noise, the radial velocity (RV) precision limit is on the order of $20 \mathrm{~m} \mathrm{~s}^{-1}$. The total error budget is discussed in Sect. 6.1.

We aimed to observe many stars with different $M / R$ ratios, to be able to precisely determine mean velocities for different groups of stars. Known binaries were excluded (M 67 is rich in these), although some single-lined spectroscopic binaries are likely to still be present. We also observed some stars with deviant velocities that appear to be non-members. The list for the whole sample, including data from Paper I, is given in the Appendix, available online.

\section{Radial velocities and membership}

Radial velocities were computed by performing a Gaussian fit to the cross-correlation function (CCF) between the observed stellar spectrum and a spectral template mask. This binary mask was based on a stellar spectrum, following the recipe of Baranne et al. (1979). As in Paper I, a K0 III synthetic mask (Baranne et al. 1996) was used for all stars, which ensured that the same reference was used for all spectra.

The mask is composed mostly of metallic lines, whose number is sufficiently high (about 2000 lines in the 500-700 nm wavelength interval used) for us to expect that line blends, which vary according to the stellar effective temperature and gravity, are symmetric and do not change the average shift of the spectrum. The cross-correlation mask is built by selecting the signal from spectral lines below a given residual flux. This cut is made as a compromise between the number of points producing the cross-correlation signal and the width of the lines: too high a threshold (including points close to the continuum) might produce too wide $\mathrm{CCFs}$, decreasing the measuring precision (see Baranne et al. 1996). For our mask, a value of 0.7 was selected, i.e., only wavelength points with a residual intensity of less than 0.7 in the K0 III reference spectrum contribute to the digital mask. This implies that the selected lines in the M 67 giants have relatively large equivalent widths.

Only a few stars have repeated observations, and the comparison between multiple measurements is shown in Fig. 1. The average radial velocity of these stars in the first observing runs, performed at the ESO $1.5-\mathrm{m}$, is $33.93 \mathrm{~km} \mathrm{~s}^{-1}$, which compares very well with $33.96 \mathrm{~km} \mathrm{~s}^{-1}$ for the same objects observed at MPG/ESO 2.2-m, although the dispersion is quite large, around $0.45 \mathrm{~km} \mathrm{~s}^{-1}$. This number is, however, an upper limit to the measurement error, because among the 14 stars observed at the two telescopes, some are probably binaries. The velocity of three stars (S1016, S1075, S1305) differ by more than $0.8 \mathrm{~km} \mathrm{~s}^{-1}$, and once these are removed from the sample, the dispersion drops to $0.3 \mathrm{~km} \mathrm{~s}^{-1}$. We consider this as a good estimate of the real uncertainty of a single measurement, though it might still be an upper limit. This value is similar to the dispersion in measurements of our RV standard star, HD 10700 (see Sects. 5.1 and 6.1.2).

Figure 2 shows the radial velocity distribution of all the observed stars. The M 67 members are clearly visible, and their RV distribution can be represented well by a Gaussian distribution centered on RV $=33.73$ with $\sigma=0.83 \mathrm{~km} \mathrm{~s}^{-1}$. We performed a $2.5 \sigma$ clipping and considered only the stars left as bona fide M67 single members. Using this selection, a total of 33 stars 


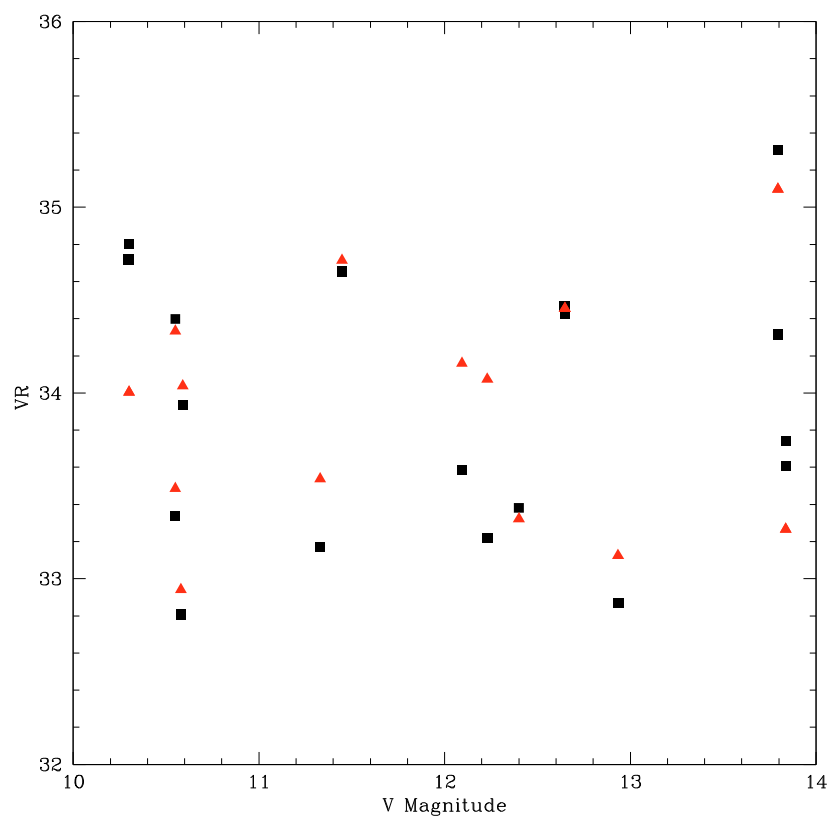

Fig. 1. Radial velocities for the 14 stars in common to the different observing runs. Observations at the ESO 1.5-m telescope are black squares; those at the MPG/ESO 2.2-m are [red] triangles. The difference between the averages from the two telescopes is less than $30 \mathrm{~m} \mathrm{~s}^{-1}$, and the spread is $0.3 \mathrm{~km} \mathrm{~s}^{-1}$ after 3 suspected binaries are discarded.

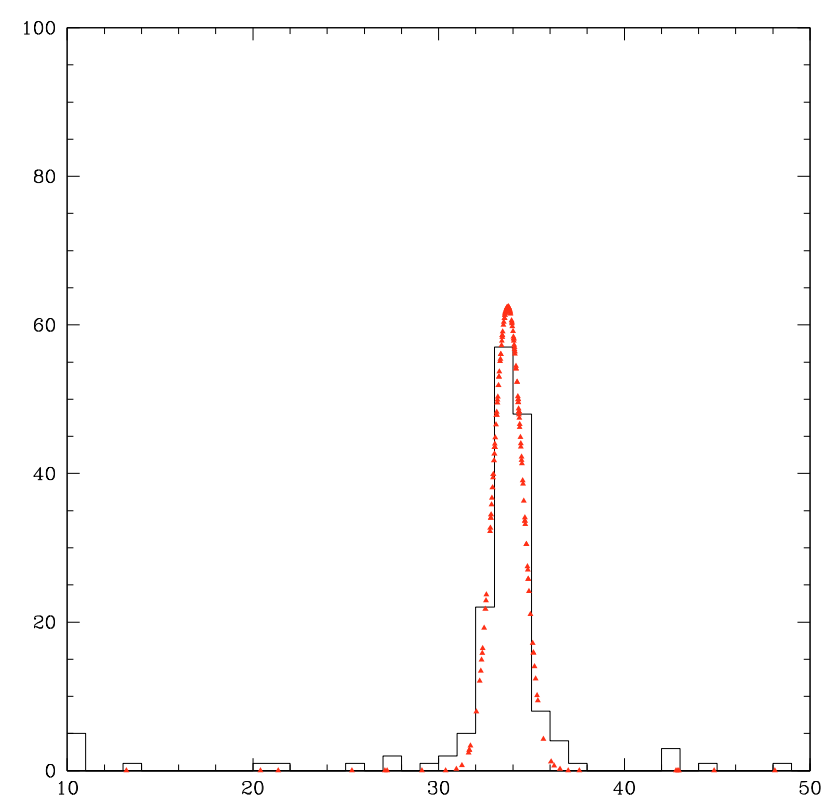

Fig. 2. Radial velocity (RV) distribution of the observed stars in M 67 (continuous line). A Gaussian distribution centered on $V r=33.73$ and with $\sigma=0.83 \mathrm{~km} \mathrm{~s}^{-1}$ is superimposed.

were discarded. We note that S1011, 1064, 1221, 1250, 1314, all classified as binaries in Paper I, appear in the list of binary stars. From Paper I and the list of Latham et al. (1992), only S986 was not recovered as a binary. The final sample consists of 110 stars and their color-magnitude diagram is given in Fig. 3; bona fide single members are marked as filled squares, stars

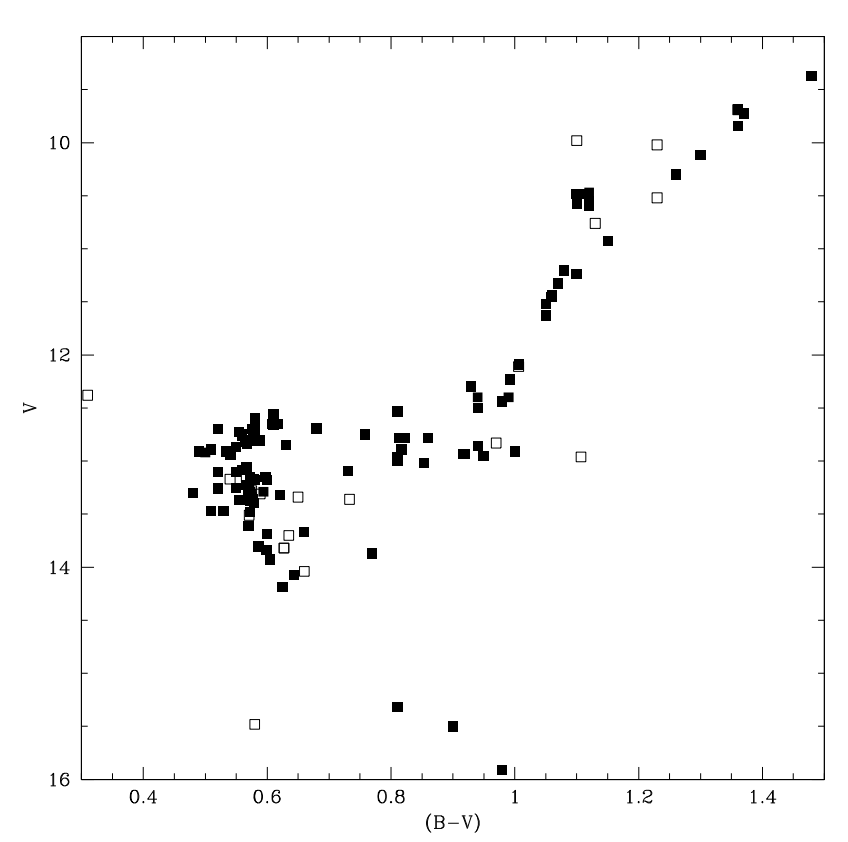

Fig. 3. M 67 color-magnitude diagram with the observed targets. Empty boxes are stars discarded on the basis of their radial velocities differing more than $2.5 \sigma$ from the average, or known to be binaries. Filled squares denote stars retained as bona fide single members.

discarded as empty squares. The targets are well distributed in the color-magnitude diagram, and numerous evolved stars were observed to ensure that good statistics could be obtained also for that less populated group.

M 67 is a well studied cluster, and the most appropriate comparison with previous radial velocity studies is probably with the pioneering work by Mathieu et al. (1986), which is similar in size to ours (104 likely single members), with comparable (but slightly worse) RV precision. A difference is that their sample was limited to brighter magnitudes, since it included all proper motion candidates brighter than $V=12.8$, which corresponds to the region around the turnoff. Those authors used (as we do) a cross-correlation technique, although their spectra were limited to a smaller spectral range, centered on $520 \mathrm{~nm}$. We analyzed the Mathieu et al. (1986) radial velocities exactly in the same way as ours, finding a mean velocity $33.57 \mathrm{~m} \mathrm{~s}^{-1}\left(\sigma=0.78 \mathrm{~km} \mathrm{~s}^{-1}\right)$ and, anticipating the results of the next section, their data also show no evidence of gravitational redshift.

\section{Analysis}

After cleaning the sample, we differentiate roughly between "main sequence" and "giants", defining as giants all stars along the subgiant and red-giant branches redder than $B-V=0.7$, and all the other stars as belonging to the main sequence. The giants' radii are so large that the gravitational redshift is expected to be negligible in these, hence their average RV should be bluer than the main-sequence stars by several hundred $\mathrm{m} \mathrm{s}^{-1}$. This simple division of the sample however does not produce the expected result, as is evident in Fig. 4:

1. In contrast to expectations from gravitational redshift predictions, the two distributions are centered on almost the same velocity $\left(33.75 \mathrm{~km} \mathrm{~s}^{-1}\right)$. 


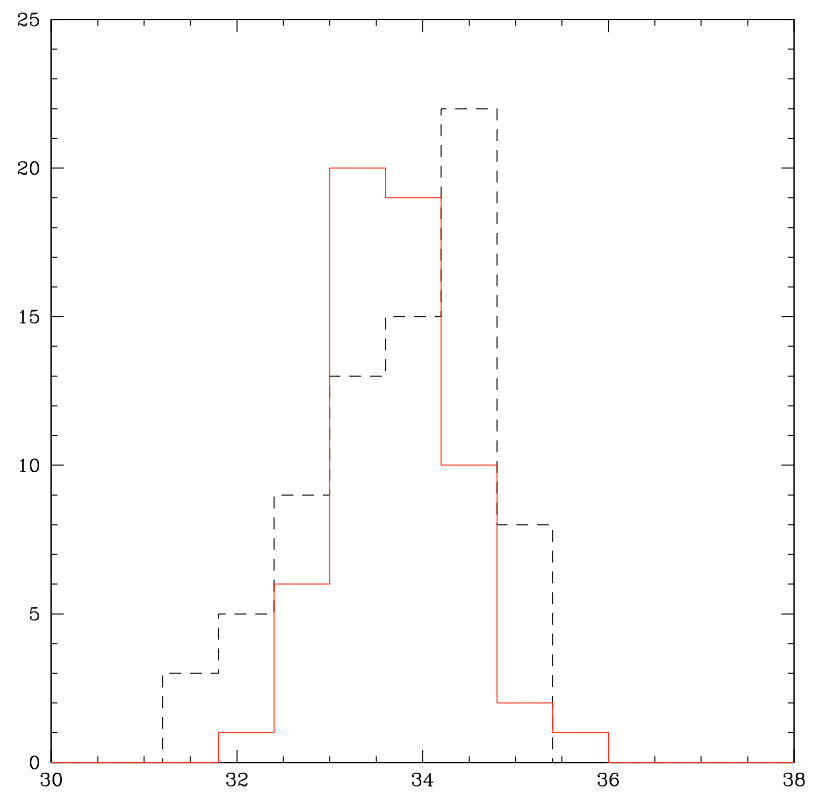

Fig. 4. Distribution of the measured radial velocities (RV) for the sample stars, sub-divided into "giant" and "dwarf" sub-samples. Giants include all evolved stars with $B-V>0.7$. No difference is present in the central value of the two distributions. The dwarf distribution (dashed black line) shows a larger spread than the giants (904 vs. $680 \mathrm{~m} \mathrm{~s}^{-1}$ )

2. Main-sequence stars and giants have substantially different velocity dispersions of $\sim 0.90$ and $0.68 \mathrm{~km} \mathrm{~s}^{-1}$, respectively.

In proceeding with a more complete analysis, masses and radii were computed for all stars. While the mass range is small, the radii vary considerably. These were computed using stellar bolometric magnitude and effective temperature, calculated from the $B-V$ versus (vs.) $T_{\text {eff relation and the bolometric magnitudes of }}$ Alonso et al. (1996, 1999). A reddening of 0.041 (An et al. 2007; Taylor 2007) and a distance modulus of 9.61 (Pasquini et al. 2008) were assumed for the cluster. For the masses, a fit between mass and luminosity was derived from evolutionary isochrones (Girardi et al. 2000) assuming solar metallicity and solar age. For the evolved stars, a flat initial mass of $1.32 M_{\odot}$ was adopted, although small imprecisions in masses or radii do not influence our conclusions.

The main result is given in Fig. 5, RV as a function of the mass-to-radius $(M / R)$ ratio. If gravitational redshift were the only mechanism acting, one would expect a linear relationship, which is not present: the overall slope of the observed data can actually be fitted with a coefficient consistent with zero.

A closer analysis suggests that a trend among the dwarfs (e.g., $M / R>0.5)$ might be present, fitted by a $R V=0.99(M / R)$ law. However, given the small range in $M / R$ (a factor 2 only) covered by the main-sequence stars, the uncertainty in this slope is large, and the slope is not statistically significant. Giants, if analyzed alone, have instead a negative slope of RV vs. $M / R$, and a large error.

\subsection{Maximum likelihood estimation of the kinematic cluster parameters}

Following the approach laid out by Pryor \& Meylan (1993), we performed a maximum likelihood estimation (MLE) of the

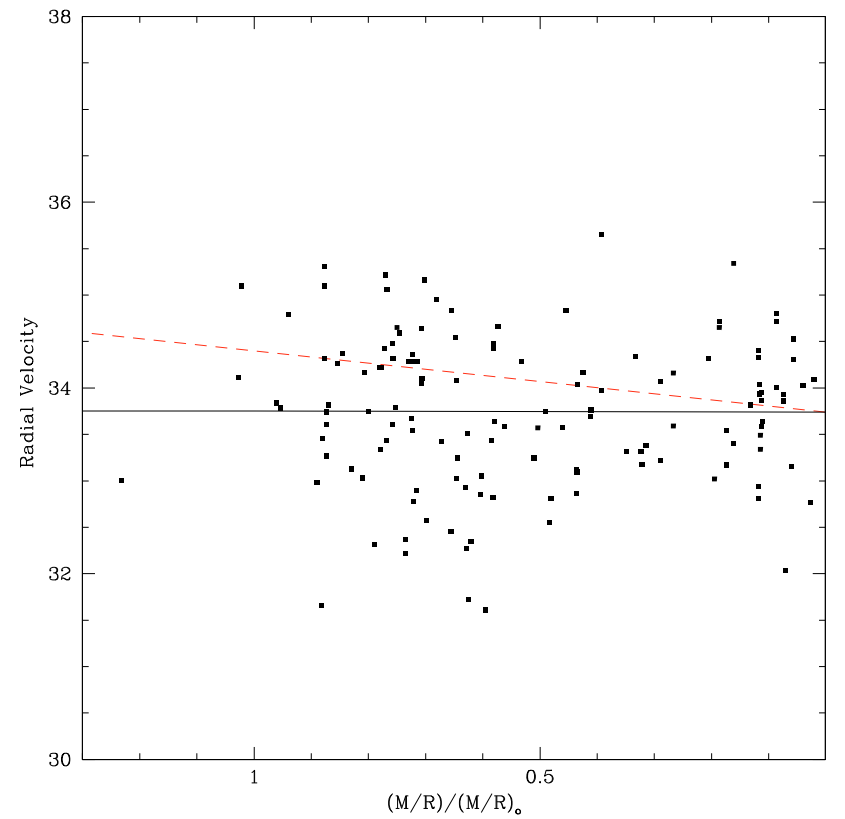

Fig. 5. Examining the gravitational redshift predictions: radial velocity vs. the mass/radius ratio $(M / R)$. The black line is the fit to the data, while the dashed [red] line shows the linear dependence upon $M / R$ expected from the gravitational redshift formula. This dependence is not seen, at least when all the stars are considered. Limiting the analysis to the "main sequence" objects $(M / R>0.5)$, a trend (though not statistically significant) might be present.

kinematic cluster parameters assuming a simple model including the effect of the gravitational redshift, and separating the two stellar groups of giants and dwarfs. We are interested in the cluster systemic velocity $v_{\mathrm{c}}$, the velocity dispersion of giants and dwarfs, $\sigma_{\mathrm{g}}$ and $\sigma_{\mathrm{d}}$, respectively, as well as the dependence of the spectroscopically measured velocity on $(M / R)$ - presumably due to the gravitational redshift. We assumed that each individual measurement $i$ was drawn from a probability density

$l_{i}\left(v_{\mathrm{c}}, \alpha, \sigma_{\mathrm{x}}^{2}\right)=\frac{1}{\sqrt{2 \pi\left(\sigma_{\mathrm{x}}^{2}+\sigma_{\mathrm{m}}^{2}\right)}} \exp \left(-\frac{\left(v_{i}-v_{\mathrm{c}}-\alpha P_{i}\right)^{2}}{2\left(\sigma_{\mathrm{x}}^{2}+\sigma_{\mathrm{m}}^{2}\right)}\right)$,

where $v_{i}$ is the spectroscopically measured radial velocity, $\sigma_{\mathrm{x}}$ the velocity dispersion of the group of stars the object $i$ belongs to, and $\sigma_{\mathrm{m}}$ the measurement error on a single star, which we assumed to be the same for all stars at $0.3 \mathrm{~km} \mathrm{~s}^{-1}$. For brevity, we introduced $P_{i} \equiv(M / R)_{i}$, where $\alpha$ is a constant of proportionality that should be $0.636 \mathrm{~km} \mathrm{~s}^{-1}$ per unit $M / R$ (in solar units) if the expected gravitational redshift was present ${ }^{1}$. We assumed that there is no measurement uncertainty in the $P_{i}$. This approximation is justified since the uncertainties are dominated by the spectroscopic ones, which correspond to an uncertainty in $P_{i}$ of $\sim 0.5$, much worse than the precision expected for $P_{i}$-values from isochrone fitting.

We maximised the overall likelihood

$L\left(v_{\mathrm{c}}, \alpha, \sigma_{\mathrm{g}}^{2}, \sigma_{\mathrm{d}}^{2}\right)=\prod_{i \in\{\text { giants }\}} l_{i}\left(v_{\mathrm{c}}, \alpha, \sigma_{\mathrm{g}}^{2}\right) \prod_{j \in\{\text { dwarfs }\}} l_{j}\left(v_{\mathrm{c}}, \alpha, \sigma_{\mathrm{d}}^{2}\right)$,

i.e., we assumed that giants and dwarfs share the same systemic velocity, and exhibit an intrinsic line shift proportional to their

${ }_{1}^{1}$ Here, we neglected the $3 \mathrm{~m} \mathrm{~s}^{-1}$ redshift in the gravitational potential of the Sun at Earth orbit. 
mass to radius ratio. Giants and dwarfs can potentially have different velocity dispersions - as expected from the previous analysis. The ansatz for the likelihood above emphasizes the role of intrinsic line shifts in the determination of the kinematic parameters of the cluster, and the need to correct for intrinsic shifts if one aims to precisely determine the kinematic properties of cluster members.

We followed the standard procedures for determining the most likely parameters and their covariance matrix, except that we used the variances instead of standard deviations as actual parameters in the minimisation process. This had mostly practical reasons since some algebraic expressions became much more compact this way. For a single group of stars with velocity dispersion $\sigma_{\mathrm{x}}$ the best-fit parameters could be found analytically as

$\alpha=\frac{\left\langle v_{i} P_{i}\right\rangle-\left\langle v_{i}\right\rangle\left\langle P_{i}\right\rangle}{\left\langle P_{i}^{2}\right\rangle-\left\langle P_{i}\right\rangle^{2}}$,

$v_{\mathrm{c}}=\left\langle v_{i}\right\rangle-\alpha\left\langle P_{i}\right\rangle$,

and

$\sigma_{\mathrm{x}}^{2}=\left\langle\left(v_{i}-v_{\mathrm{c}}-\alpha P_{i}\right)^{2}\right\rangle-\sigma_{\mathrm{m}}^{2}$,

where $\langle$.$\rangle denotes the (ensemble) average. We did not find an$ analytical solution for the case of two different velocity dispersions. In this case, we solved the minimisation problem numerically by direct iteration on $\sigma_{\mathrm{g}}^{2}$ and $\sigma_{\mathrm{d}}^{2}$ as suggested by Pryor \& Meylan (1993). With the best-fit parameters, we evaluated the covariance matrix $\left(-\partial^{2} L / \partial \theta_{i} \partial \theta_{j}\right)^{-1}$, where $\theta$ is the vector of parameters $\theta=\left(v_{\mathrm{c}}, \alpha, \sigma_{\mathrm{g}}^{2}, \sigma_{\mathrm{d}}^{2}\right)$. We obtained the best-fit parameters and their $1 \sigma$ uncertainties as

$v_{\mathrm{c}}=33.824 \pm 0.121 \mathrm{~km} \mathrm{~s}^{-1}$
$\alpha=-0.134 \pm 0.230 \mathrm{~km} \mathrm{~s}^{-1}$
$\sigma_{\mathrm{g}}=0.566 \pm 0.068 \mathrm{~km} \mathrm{~s}^{-1}$
$\sigma_{\mathrm{d}}=0.849 \pm 0.078 \mathrm{~km} \mathrm{~s}^{-1}$.

The errors in the standard deviations were calculated from the errors in the variances by applying the standard error propagation formula. From the covariance matrix, we calculated the correlation coefficients among parameter pairs: $-0.840\left(v_{\mathrm{c}}, \alpha\right)$; $-0.067\left(v_{\mathrm{c}}, \sigma_{\mathrm{g}}^{2}\right) ; 0.059\left(v_{\mathrm{c}}, \sigma_{\mathrm{d}}^{2}\right) ; 0.093\left(\alpha, \sigma_{\mathrm{g}}^{2}\right) ;-0.081\left(\alpha, \sigma_{\mathrm{d}}^{2}\right)$; and $-0.008\left(\sigma_{\mathrm{g}}^{2}, \sigma_{\mathrm{d}}^{2}\right)$. The only significant and actually strong correlation among the parameters is the one between $v_{\mathrm{c}}$ and $\alpha$. This is understandable since changes in the systemic velocity can to some extent be traded against changes in the intrinsic line shifts. The velocity dispersions in each group of stars are largely insensitive to the systemic velocity and the intrinsic line shifts. All in all, the MLE analysis confirms our findings that there is a clear difference $(3.6 \sigma)$ between the velocity dispersions of giants and dwarfs, and a dependence of the intrinsic line shifts on $(M / R)$, which deviates significantly $(3.3 \sigma)$ from the expected behaviour for a signal solely because of a gravitational redshift.

\section{Discussion}

Two main results can be derived from our data: no obvious signature of gravitational redshift is seen in the data, and the dispersion of the radial velocities is higher in un-evolved stars than in evolved ones. We discuss these two results separately.

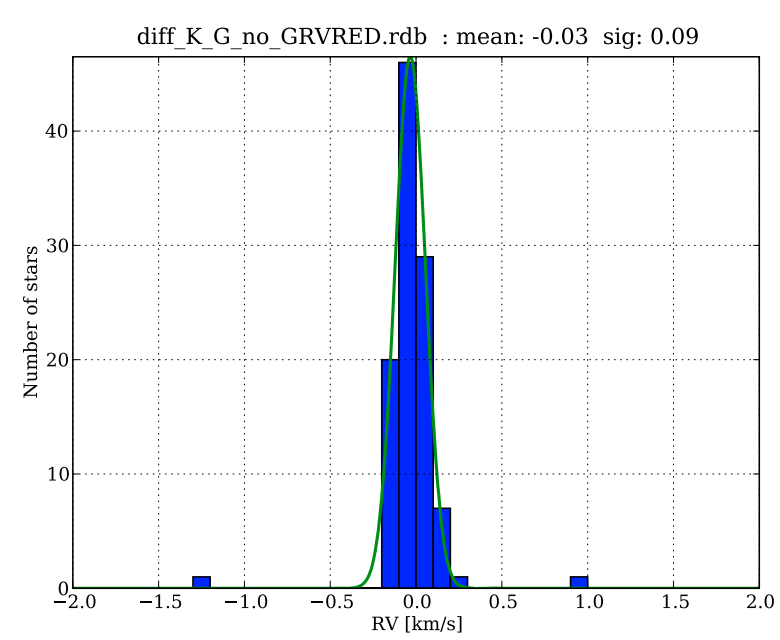

Fig. 6. Histogram of the differences of radial velocities obtained by using two different cross-correlation masks (K0 III giant and G2 V dwarf): $R V_{\mathrm{K}}-R V_{\mathrm{G}}$. The Gaussian fit gives a mean of $-30 \mathrm{~m} \mathrm{~s}^{-1}$ and an rms of $90 \mathrm{~m} \mathrm{~s}^{-1}$.

\subsection{Missing gravitational redshift}

As discussed above, a radial velocity shift of up to $600 \mathrm{~m} \mathrm{~s}^{-1}$ is expected between main sequence stars and giants. This difference is not present in our data as illustrated in Figs. 4 and 5. Could we have missed the gravitational redshift because of some systematic effect?

\subsubsection{Template effects}

Given that the mask used is of spectral type K0 III, a better match between template and target star is expected for the sub-giants and giants than for main-sequence stars. One clear effect of using the K0 III mask with G-type turn-off stars is that the resulting CCF is shallower than for the giants and sub-giants (Fig. 3 of Paper I). This is because of both the mismatch between star and template, and the generally weaker lines in dwarf stars; the depth of the CCF is thus related to the luminosity.

Could the mismatch between luminosity class and mask have caused a velocity shift in the turnoff stars? To investigate this, we computed new cross-correlation functions for all stars using a G2 V mask instead. The mean RV difference was found to be a well defined value $\left(30 \mathrm{~m} \mathrm{~s}^{-1}\right)$ caused by template zeropoint mismatch. The RV difference distribution has an rms of $120 \mathrm{~m} \mathrm{~s}^{-1}$. A detailed inspection of spectra finds differences larger than $100 \mathrm{~m} \mathrm{~s}^{-1}$ and that low $S / N$ along with a non optimum cosmic-ray cleaning are responsible. In addition, most of the discrepant spectra were recorded during the last season, when a simultaneous Th-Ar lamp was used, contaminating faint spectra through its emission lines. If only data from the first two seasons are considered, the rms drops to $90 \mathrm{~m} \mathrm{~s}^{-1}$, as in Fig. 6 .

While these tests show some limits in the precision, the use of a different mask also shows that there is no offset introduced by the choice of the mask differentially affecting various groups of stars. However, an extra error of about $100 \mathrm{~m} \mathrm{~s}^{-1}$ due to poor cosmic-ray cleaning and ThAr contamination has to be added to the formal error of the cross-correlation fit.

We also tested different mask characteristics, such as the dependence of the measured RV upon the wavelength range, finding that values retrieved from the $500-700 \mathrm{~nm}$ mask are within a few tens of $\mathrm{m} \mathrm{s}^{-1}$ from those found for the "full" 400-1000 nm 


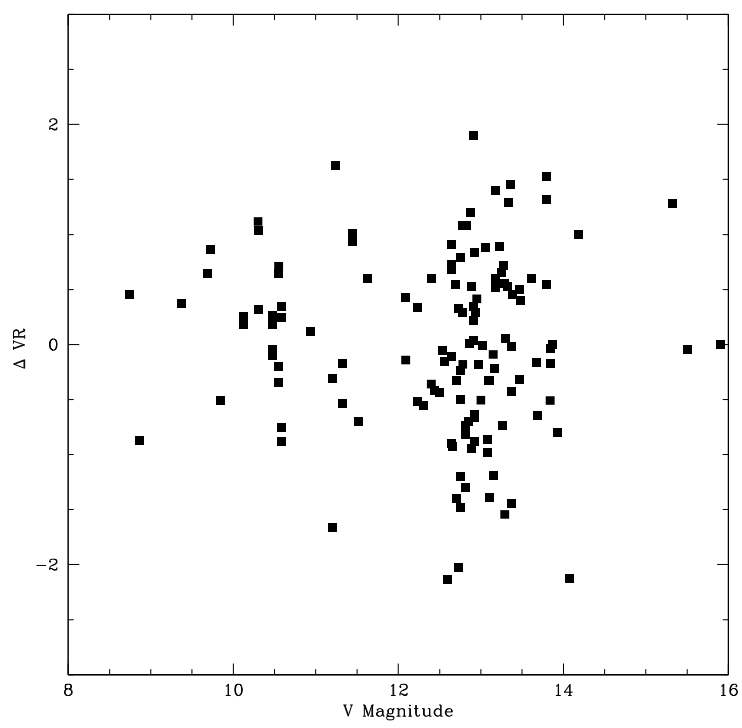

Fig. 7. Residuals of measured radial velocity VR as function of stellar apparent magnitude. The residuals show a typical increase with magnitude as expected from the greater noise of fainter stars. The effect might also be due to a dynamically hotter population of dwarfs, or to some observational bias.

one, for different types of stars. We therefore conclude that the mask and wavelength range used do not induce systematic effects that could cancel the gravitational redshift signal.

\subsubsection{Spectrograph drifts}

Variations in temperature and atmospheric pressure during the observing night can cause spectral drifts of a few hundred $\mathrm{m} \mathrm{s}^{-1}$ (e.g., Platais et al. 2007). This effect is seen in our data collected in the third season, where the simultaneous calibration fiber was used. Unfortunately, the first two datasets did not make use of the simultaneous calibration fiber and the spectrograph drifts could not be removed.

To estimate the amplitude of the drifts caused by changes in atmospheric conditions, we used 52 observations of $\tau$ Cet (HD 10700) taken from the SACY survey (Torres et al. 2006) and from our own observations. These spectra were reduced and analyzed in a similar way to the present M 67 stars. They cover a time span of 3000 days from Oct. 1999 to Feb. 2008. The mean $\mathrm{RV}$ is $-16.37 \mathrm{~km} \mathrm{~s}^{-1}$ with an rms of $0.3 \mathrm{~km} \mathrm{~s}^{-1}$. The best fit, excluding 6 extreme values, gives a similar mean, and an rms of $0.18 \mathrm{~km} \mathrm{~s}^{-1}$. Thus we believe that the drift error is about $200 \mathrm{~m} \mathrm{~s}^{-1}$.

Simulations of the effect of photon noise in the RV computation show that with a $S / N$ as low as 10-30, radial velocity errors are as low as a few tens of $\mathrm{m} \mathrm{s}^{-1}$ (Paper I; Bouchy et al. 2001). However, those simulations only consider white (photon) noise, and (as discussed above) there are additional sources of errors associated with low $S / N$. In Fig. 7, we plot the scatter diagram as a function of stellar magnitude and indeed the behaviour is that expected, if the noise was not negligible.

We investigate whether one can reproduce the missing gravitational trend and the scatter seen in Fig. 7 by accounting for the different sources of spread in the VR. First, the intrinsic velocity dispersion of the cluster of $0.8 \mathrm{~km} \mathrm{~s}^{-1}$ was considered. A Gaussian drift error drawn from a distribution with an rms of

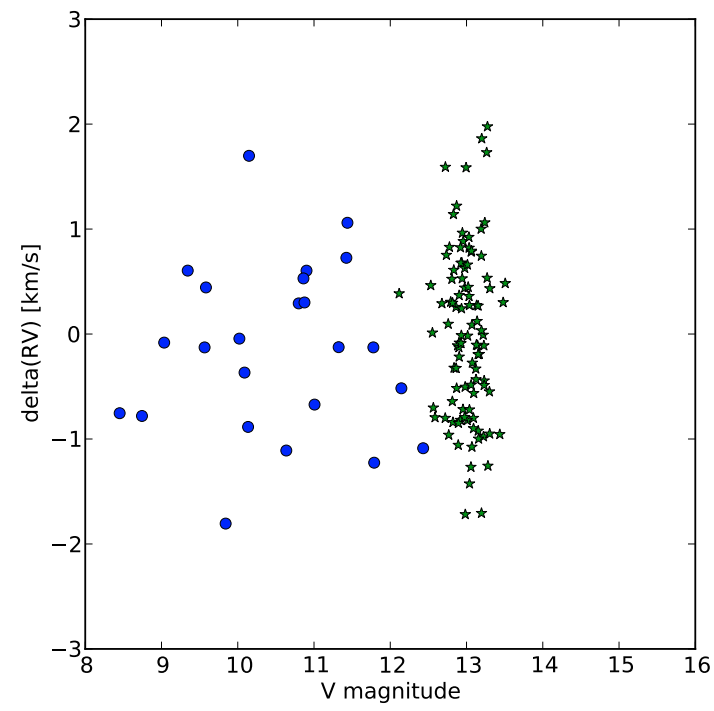

Fig. 8. Synthetic reproduction of the observed residual RV vs. magnitude diagram of Fig. 7. As input for the points, our various sources of noise were used, together with the velocity dispersion inside the cluster.

$200 \mathrm{~m} \mathrm{~s}^{-1}$ and a photon noise of $150 \mathrm{~m} \mathrm{~s}^{-1}$ for a $V=14$ star were added. This noise was scaled as the square root of the flux for a given magnitude (i.e., $(S / N)^{-1}$, assuming that photon noise is the limiting factor for faint targets).

We then computed the difference between the centers of the $\mathrm{RV}$ distribution of main sequence and giant stars, which is always below $100 \mathrm{~m} \mathrm{~s}^{-1}$. Moreover, the rms of both distributions is clearly dominated by the cluster velocity dispersion. An additional gravitational shift of $600 \mathrm{~m} \mathrm{~s}^{-1}$ added to the distribution of sub-giant and giant stars can be retrieved in all cases in spite of their limited number.

We have so far been unable to identify any instrumental or observational effect that could cancel out the expected gravitational redshift signal, and we conclude that our observations find no evidence of a gravitational redshift trend.

Nordström et al. (1997) briefly discuss a similar case, because they found a difference of $0.4 \mathrm{~km} \mathrm{~s}^{-1}$ (dwarfs redder than giants) between the average $\mathrm{RV}$ of giants and main sequence stars in the open cluster NGC 3680, almost exactly that predicted from the gravitational redshift. NGC 3680 is an almost completely evaporated cluster, with only a few bona-fide members left. The paucity of the sample precludes certain conclusions, but could suggest that our results about M 67 may not be generalized to other clusters. On the other hand, that no gravitational redshift is found by analyzing the published data by Mathieu et al. (1986) shows that this result is not limited to our observations only, but is a characteristic of this cluster.

\subsubsection{Cluster dynamics}

Even if open clusters are not known to rotate or to have complex dynamical topologies, we analyzed whether the missing gravitational signature could be due to a combination of complex cluster dynamics and bias selection, namely that the observed giants and dwarfs are situated in different spatial regions of the cluster, perhaps affected by different global velocities. In this case, the stars would not share the same systemic velocity and this bias could conspire to cancel the gravitational redshift signature. For 

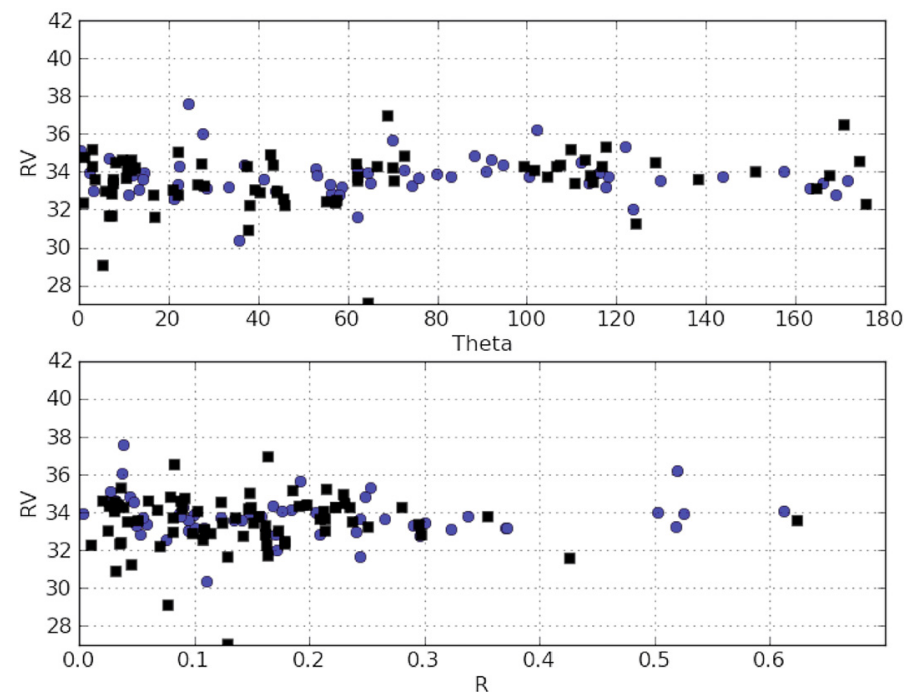

Fig. 9. Angular and radial distribution of measured radial velocities (black squares denote giants; [blue] dots are main sequence stars). No evidence of complex dynamics or dynamical heating at larger distances is observed.

M 67, there is no report of complex dynamics, with the exception of a claim by Loktin et al. (2005) of a possible expansion of the cluster core. No evidence of rotation is found in the analysis of the Mathieu et al. (1986) data by the same authors. To investigate effects related to cluster dynamics, we analyzed the velocity distribution of the observed stars as a function of $R$ (distance from the cluster center) and $\theta$ (angular position) of giants and dwarfs, finding no evidence of RV variations with these parameters (Fig. 9). We note in passing that we do not find evidence for a larger dispersion at large radii. We therefore conclude that complex cluster dynamics coupled to a selection bias cannot explain the non-detection of gravitational redshift.

\subsubsection{Convective shifts}

Dynamic effects in stellar atmospheres may produce net shifts in photospheric absorption lines, and we cannot exclude that lines in main-sequence stars could be more blueshifted than in giants, thus (partially) canceling the expected gravitational redshift signature.

This differential shift need not be present in all spectral lines, but only (in a statistical sense) in the deeper ones. For a given metallicity, typical lines (metallic neutral ions) are generally deeper in cool giants than in dwarfs. By using the same mask for all stars, we use lines with different equivalent widths in the two classes of stars, which are relatively deeper in the giant spectra.

Since single spectra of each star have a rather low $S / N$, we constructed two "average" spectra from two sets of low $S / N$ spectra: one from 64 selected dwarfs (called "dwarf") and one for 20 selected giants ("giant"). The spectra were first normalized in flux at $600 \mathrm{~nm}$, and then averaged by calculating the medians. Given the low velocity dispersion of the cluster stars, these co-added spectra are not appreciably broadened. The $S / N$ of these spectra is about 200 in the region of interest for the "dwarf" spectrum and about 140 for the "giant" one. The coadded spectra still show - within the uncertainties - the same radial velocity for "giant" and "dwarf" spectra alike. We selected a number of lines used for chemical analysis of solar metallicity stars (Pasquini et al. 2004), inspecting them visually in the coadded spectra. From the original list, 52 lines passed this quality

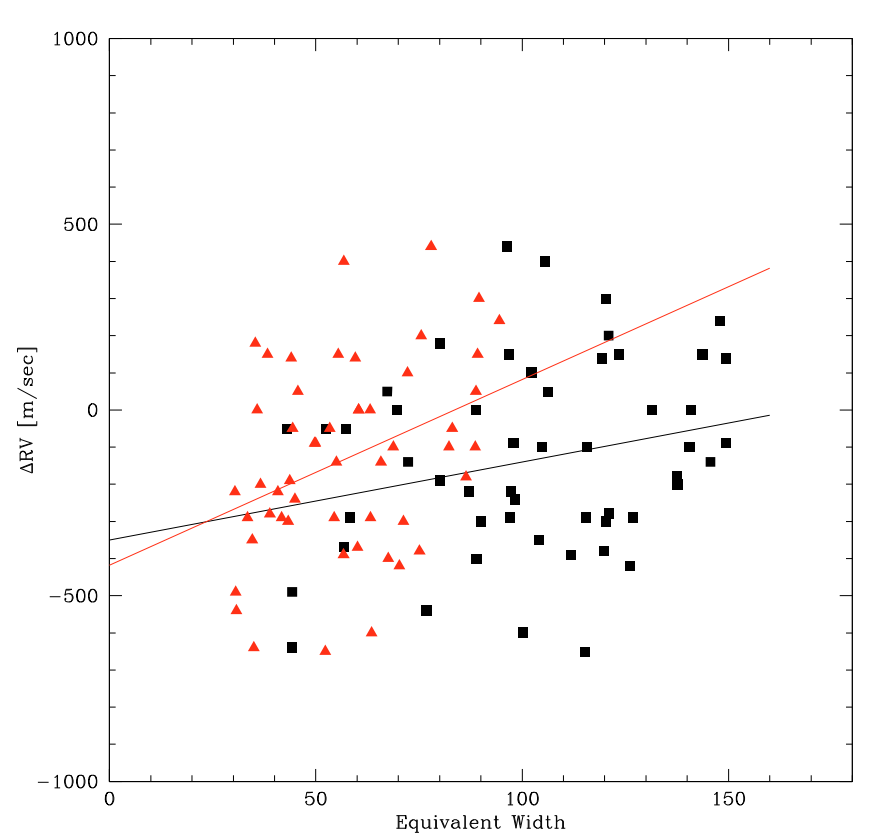

Fig. 10. Difference between measured radial velocities of selected lines in the median "giant" (black squares) and "dwarf" spectra ([red] trian-

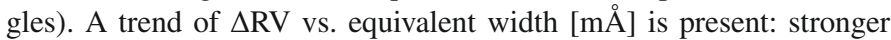
lines show smaller (negative) differences between dwarfs and giants. For strong lines, the expected gravitational redshift probably is canceled by atmospheric dynamical effects.

check, and were fitted with a Gaussian function, using the ARES package (Sousa et al. 2008). We first tested with the IRAF task SPLOT the consistency between the automatic program and the manual fitting. The central wavelengths of the "dwarf" and "giant" spectra were recorded, and the $D V=V_{\text {giant }}-V_{\text {dwarf }}$ is plotted in Fig. 10 as a function of line equivalent widths. For "giant" lines weaker than about $4 \mathrm{pm}(40 \mathrm{~m} \AA)$, the measurements were not very reliable, and these weak lines are not used. On the other hand, lines with equivalent widths above $15 \mathrm{pm}(150 \mathrm{~m} \AA)$ were discarded to minimize possible contamination by blends. In both plots of Fig. 10, a trend is present: the stronger the line, the redder the shift in the giant spectrum. The observed relative shifts are up to $\pm 500 \mathrm{~m} \mathrm{~s}^{-1}$, comparable to the expected gravitational redshift. We looked for, but did not find any dependence on other quantities (e.g., wavelength).

Formal fits to the trends indicate: $\mathrm{D} V=$ $2.1 E W_{\text {giants }}-350 \mathrm{~ms}^{-1}$ and $\mathrm{DV}=5.0 E W_{\mathrm{dwarfs}}-418 \mathrm{~ms}^{-1}$. The trends are only marginally significant (correlation coefficients 0.25 and 0.35 ). The formal error in measurements of single lines is about $50 \mathrm{~ms}^{-1}$, and we believe that the observed spread is real. The lines used originate mostly in neutral elements (dominated by $\mathrm{Fe}$ ) with different characteristics, but also a few Fe II lines are used. With higher $S / N$ spectra for single stars, it would be possible to investigate trends between different chemical elements and line parameters, but we believe that for our current data this would be too ambitious.

Wavelength shifts induced by convective motions have been studied in particular for lines in the Sun and in Procyon, where it has been possible to both measure and model intrinsic lineshapes using very high spectral resolution (Dravins 1982; Allende Prieto et al. 2002a); a successively larger blueshift for weaker lines is a signature of the three-dimensional photospheric 
hydrodynamics (e.g., Asplund et al. 2000). In other cool stars, Allende Prieto et al. (2002b) and Ramírez et al. (2008) also observed how lines from neutral species get more blueshifted as they become fainter.

Since we are able to compare dwarfs and giants of the same cluster, we can also see that the trend seems to differ between main-sequence and evolved stars. For the M67 evolved stars, the blueshift is less than in turnoff ones, and the expected gravitational redshift is seen only for a few of the faintest lines. The blueshift variation with equivalent width is larger in giants than in dwarfs, as suggested by the trend in Fig. 10. A caveat, however, is that additional parameters may be important, for instance line broadening by stellar rotation, which differs between dwarfs and giants: rotational broadening changes line asymmetries and may affect the velocity signal measured by cross-correlation (Dravins \& Nordlund 1990).

This discussion suggests a plausible explanation for our nondetection: gravitational redshift is largely canceled because our correlation mask is dominated by rather deep lines, which are more blueshifted in main-sequence stars than in giants because of photospheric convective motions.

\subsection{Convective line shifts in $3 D$ model atmospheres}

To investigate the apparent absence of gravitational redshift, synthetic spectral lines were computed from $3 \mathrm{D}$ and time-dependent model atmospheres. We synthesized 15 artificial Fe I lines, spanning the range of excitation potential and equivalent width of the observed ones. All lines were placed at a wavelength of $620 \mathrm{~nm}$, which is the average of the observed Fe I lines in the range 586$672.7 \mathrm{~nm}$. The 3D model atmospheres providing the thermal and kinematic information for the spectral synthesis were taken from the CFIST 3D model atmosphere grid (Ludwig et al. 2009). To represent a "dwarf" and "giant", we chose for each a single 3D model atmosphere. Table 2 summarizes their basic properties.

The wavelength shifts of the synthesized lines were measured by applying the same procedure as for the observed ones, i.e., by fitting a Gaussian profile. In contrast to the observations, the artificial lines were available at very high spectral resolution $\left(\approx 2 \times 10^{6}\right)$ and $S / N$ so that their shifts with respect to the laboratory wavelength could be calculated with high fidelity. For the 32 observed Fe I lines, we calculated convective line shifts by interpolating in the grid of synthetic lines according to the observed equivalent width and excitation potential of the line. The typical statistical uncertainty to which the wavelength position of an individual line is predicted is (from previous experience) $30 \mathrm{~m} \mathrm{~s}^{-1}$. This means that for the difference between the wavelength shift of the same line between two models we expect a typical precision of $42 \mathrm{~m} \mathrm{~s}^{-1}$. The statistical uncertainty is related to issues such as how well the acoustic oscillations present in the computational box are averaged out. The example illustrates that we expect the line shifts among lines of the same model to be correlated, hence we do not expect the uncertainty in the predicted shifts among giant and dwarf to drop as the square-root of the number of lines used to characterise it. Thus, we expect a modeling uncertainty of about $\pm 40 \mathrm{~m} \mathrm{~s}^{-1}$ for the line shift difference between the giant and dwarf - not a large value but also not completely insignificant.

Figure 11 shows the predicted convective shifts in dwarfs and giants. Before determining the line shift, the synthetic spectral line profiles were broadened with a Gaussian profile of $\sigma=3.9 \mathrm{~km} \mathrm{~s}^{-1}$ which corresponds to the spectrograph resolution, combined with the macroturbulence-like velocity dispersion stemming from the motions of individual cluster stars. In

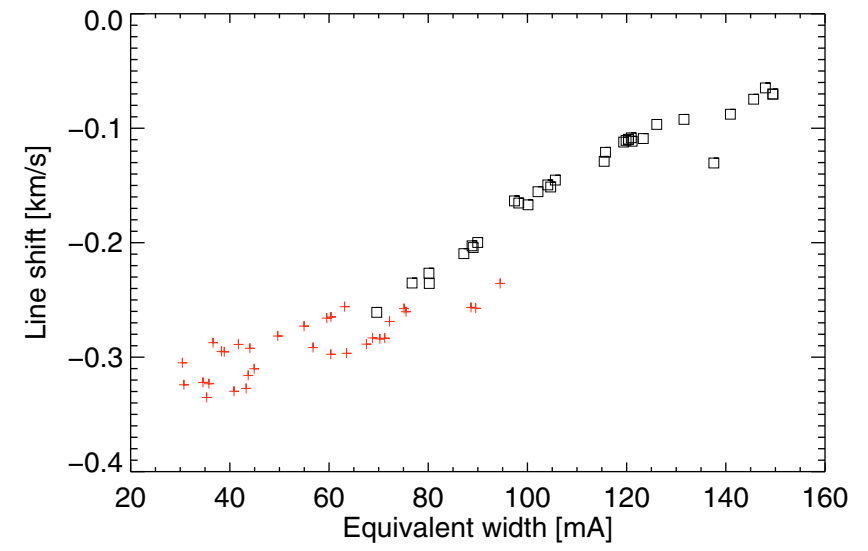

Fig. 11. Calculated convective wavelength shifts for the 32 studied Fe I lines for the dwarf (crosses) and giant (squares) models. Negative velocities correspond to absolute blueshifts (gravitational redshifts are left out).

addition, it was assumed that the dwarf rotates with a projected rotational velocity of $5 \mathrm{~km} \mathrm{~s}^{-1}$, while the giant exhibits no rotation. We find for the difference in the shifts of lines between the giant and dwarf a minimum of 62 a maximum of 211 and an average of $146 \mathrm{~m} \mathrm{~s}^{-1}$. We note that the correlation between the strengths of lines in the giant and dwarf is not very tight. However, as seen in Fig. 11, we find a trend in the convective shift with equivalent width that is continuous between dwarfs and giants: the stronger Fe I lines are on average less blueshifted in the giant.

Figure 12 depicts the calculated differences in the shift of the same line between dwarf and giant, comparing them to observations. For the synthetic lines, assumptions about spectrograph resolution and stellar rotation were the same as before. An average gravitational redshift was included, so that the giant synthetic points were blueshifted by $382 \mathrm{~m} \mathrm{~s}^{-1}$ (applying an average $M / R$ of 0.73 for dwarfs and 0.13 for giants, as for the stars used to compute the average spectra). The agreement with the observations is now quite good, with a difference of only $\sim 140 \mathrm{~m} \mathrm{~s}^{-1}$ between the synthetic and the observed average shifts.

However, the observed spectra have properties that are not predicted by the modeling, i.e. a dependence of the shift on the equivalent width and a considerable spread in the measurements. Nevertheless, we conclude that convective line shifts are the most likely explanation of our failure to observe a gravitational redshift signature.

Among the possible causes of the remaining discrepancies between models and observations, we note that the chosen $3 \mathrm{D}$ models may not be optimal representatives of the "dwarf" and "giant" spectra. Their choice was dictated by availability. For example, the iron abundance determined from the dwarf model is approximately solar but for the giant is 0.5 dex higher. This may indicate that the giant model is hotter than the typical observed giant. For the dwarfs, solar temperature is appropriate for our fainter stars, while higher temperatures are expected for the stars closer to the turnoff. Indeed a similar Fe abundance is obtained by adopting $6000 \mathrm{~K}$ and $4600 \mathrm{~K}$ for dwarfs and giants, respectively (using 1D models). However, we do not expect the line shifts to depend sensitively on stellar parameters (as long as the line strengths are matched), so we argue that our approach should give a reasonably accurate estimate of the expected lineshift differences. 
Table 2. 3D radiation-hydrodynamics model atmospheres.

\begin{tabular}{lllllllllllll}
\hline \hline Model & $\begin{array}{l}T_{\text {eff }} \\
{[\mathrm{K}]}\end{array}$ & $\begin{array}{l}\log g \\
{\left[\mathrm{~cm} / \mathrm{s}^{2}\right]}\end{array}$ & {$[\mathrm{Fe} / \mathrm{H}]$} & $\begin{array}{l}l_{\mathrm{x}}, l_{\mathrm{y}} \\
{[\mathrm{Mm}]}\end{array}$ & $\begin{array}{l}l_{\mathrm{z}} \\
{[\mathrm{Mm}]}\end{array}$ & $N_{\mathrm{x}}, N_{\mathrm{y}}$ & $N_{\mathrm{z}}$ & $N_{\mathrm{obm}}$ & $\begin{array}{l}T \\
{[\mathrm{~h}]}\end{array}$ & $\begin{array}{l}\delta I_{\mathrm{rms}} / I \\
\%\end{array}$ & $\begin{array}{l}v_{\mathrm{rms}} \\
{\left[\mathrm{km} \mathrm{s}^{-1}\right]}\end{array}$ & Modelcode \\
\hline dwarf & 5782 & 4.44 & 0.0 & 5.6 & 2.27 & 140 & 150 & 12 & 1.2 & 14.4 & 2.24 & $\mathrm{~d} 3 \mathrm{gt} 57 \mathrm{~g} 44 \mathrm{n} 58$ \\
giant & 4968 & 2.50 & 0.0 & 573 & 243 & 160 & 200 & 5 & 152 & 18.9 & 3.04 & $\mathrm{~d} 3 \mathrm{t} 50 \mathrm{~g} 25 \mathrm{~mm} 00 \mathrm{n} 01$ \\
\hline
\end{tabular}

Notes. "Model" is the model's name used in this paper, $T_{\text {eff }}$ the effective temperature, $\log g$ the gravitational acceleration, $[\mathrm{Fe} / \mathrm{H}]$ the metallicity, $l_{\mathrm{x}}=l_{\mathrm{y}}$ the linear horizontal size of the square-shaped computational box, $l_{\mathrm{z}}$ its vertical extent, $N_{\mathrm{x}}=N_{\mathrm{y}}$ the number of grid points in the horizontal directions, $N_{\mathrm{z}}$ the number in vertical direction, $T$ the duration of the simulated time series, $N_{\mathrm{obm}}$ the number of equivalent frequency points considered in the solution of the radiative transfer equation, $\delta I_{\mathrm{rms}} / I$ the relative spatial white light intensity contrast at stellar disk center, $v_{\mathrm{rms}}$ the temporal and spatial average of the vertical velocity at Rosseland optical depth unity, "Modelcode" is an internal identifier of the model sequence.

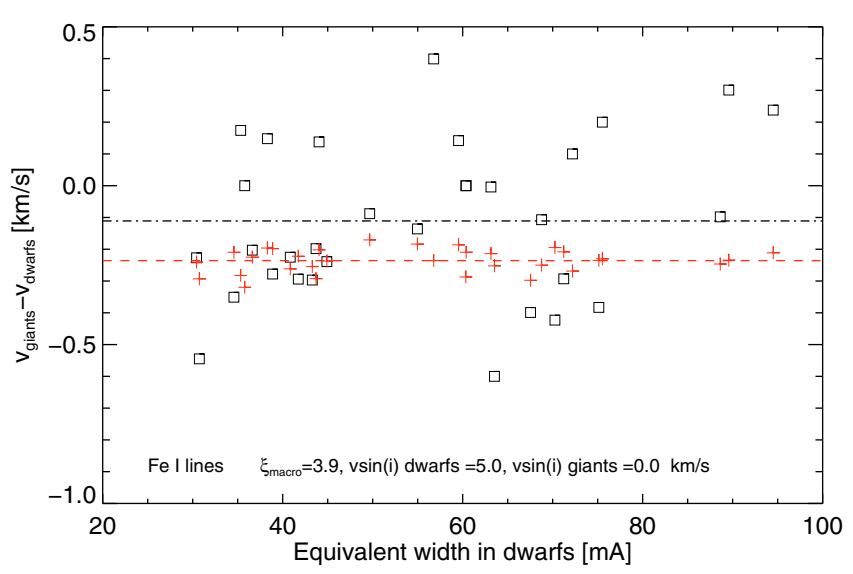

Fig. 12. Comparison of observations to synthetic wavelength shifts of Fe I lines, computed from 3D hydrodynamic models for dwarfs and giants. The computed differences between the lineshifts in giant and dwarf models is shown as crosses, and the observations as squares. The calculated shifts include the average expected contribution of the gravitational redshift of $382 \mathrm{~m} \mathrm{~s}^{-1}$.

Another possibility is the presence of noise and biases in our combined spectra; to test this idea we repeated the analysis using the Kitt Peak solar flux spectrum (Kurucz et al. 1984) and the Arcturus spectrum (Bagnulo et al. 2003). Although here we can only test the slope and the spread around the relationship, this gives qualitatively similar results to $\mathrm{M} 67$, although the slope is shallower $\left(\sim 2 \mathrm{~m} \mathrm{~s}^{-1} / \mathrm{m \AA}\right.$ instead of 5) and the scatter is smaller $\left(100 \mathrm{~m} \mathrm{~s}^{-1}\right.$ instead of $\left.240 \mathrm{~m} \mathrm{~s}^{-1}\right)$.

Being the first attempt of such a test, we believe it is encouraging. For higher $S / N$ data it will be possible to test other effects, such as the dependence of the line shifts on other parameters (element, excitation potential, ionization, wavelength). One could also apply the same technique to clusters of different metallicity and chemical composition, and to work out comparison grids for theoretical models.

\subsection{Dynamically hot dwarfs}

The observation that dwarfs are dynamically hotter than giants might seem counter-intuitive, because giants are known to be intrinsic RV variables, with variations of up to a few hundreds $\mathrm{m} \mathrm{s}^{-1}$ (e.g., Setiawan et al. 2004), and their intrinsic RV variability will add to their RV dispersion. In addition, main-sequence stars have lower masses, and in the case of mass segregation the giants should be (as they are in M67) mostly concentrated in the middle of the cluster, near the center of the potential well.
On the other hand, in an equipartition regime, giants should be dynamically cooler, because they are more massive.

The proper-motion dispersion of the bright M 67 stars measured by Girard et al. (1989) is almost 0.2 mas/yr. At a distance of $836 \mathrm{pc}$ (Pasquini et al. 2008), it corresponds to a radial velocity dispersion of $0.8 \mathrm{~km} \mathrm{~s}^{-1}$, comparing very well with the dispersion of $\mathrm{RV}=0.83 \mathrm{~km} \mathrm{~s}^{-1}$ measured for all stars and also with the $0.68 \mathrm{~km} \mathrm{~s}^{-1}$ dispersion for the giants only. The comparison of the radial velocity distributions for both populations in Fig. 4 suggests that the dwarfs are dynamically hotter than the giants in M67, i.e., they have a larger velocity dispersion. To check whether this difference might originate in some observational bias, we performed the following test: we again simulated the radial velocity distribution for the dwarfs using the same error parameters and a population of 100 stars as in Fig. 8. The rms of this artificial distribution was then computed, and the procedure repeated 5000 times. The result is a distribution of rms, centered on the velocity dispersion of the cluster $\left(800 \mathrm{~m} \mathrm{~s}^{-1}\right)$ with values between 0.6 and $1.0 \mathrm{~km} \mathrm{~s}^{-1}$. In the simulations, if we now turn off the spectrometer drift error (Sect. 6.1.2), while maintaining all other parameters, we end up with similar figures. This already indicates that the observed velocity dispersion does not depend on measurement errors, but rather on the number of objects.

As a further test, we set the drift error to its initial value and generated the rms distributions for two populations: one with $N=100$ members (corresponding to our real case) and another with 1000 members. The result is given in Fig. 13. With 1000 members, the velocity dispersion is well defined with a central value of $0.8 \mathrm{~km} \mathrm{~s}^{-1}$ and 0.73 to $0.88 \mathrm{~km} \mathrm{~s}^{-1}$ peak-to-peak. Therefore, the observation that the M 67 dwarfs have a larger velocity dispersion than the giants does not seem to be caused by our measurement errors but is possibly an effect of low number statistics.

We note that Nordström et al. (1997) found a similar result for NGC 3680, with the dwarfs showing a dispersion of $0.67 \mathrm{~km} \mathrm{~s}^{-1}$ compared to 0.36 for the giants. Our analysis of the Mathieu et al. (1986) data (which, however, contain a limited number of main-sequence stars) is in perfect agreement with our findings, giving $\sigma(R V)=0.89$ and $0.66 \mathrm{~km} \mathrm{~s}^{-1}$ for dwarfs and giants, respectively.

Since we cannot exclude that the effect is real, we note that a similar result was found by Zhao et al. (1996) and Loktin et al. (2005) by analyzing proper motion data. Zhao et al. (1996), analyzing a sample of about 200 stars, found that the dispersion in the proper motions of M 67 bright stars $(V<13.5)$ is significantly smaller than that of fainter objects $(13.5<V<14.5)$, by a factor $\sim 1.3$. They also found that the brighter (more massive) stars are more concentrated towards the center of the cluster. Loktin et al. (2005) found a similar result, with a much larger sample. The larger sample allows them to subdivide the 


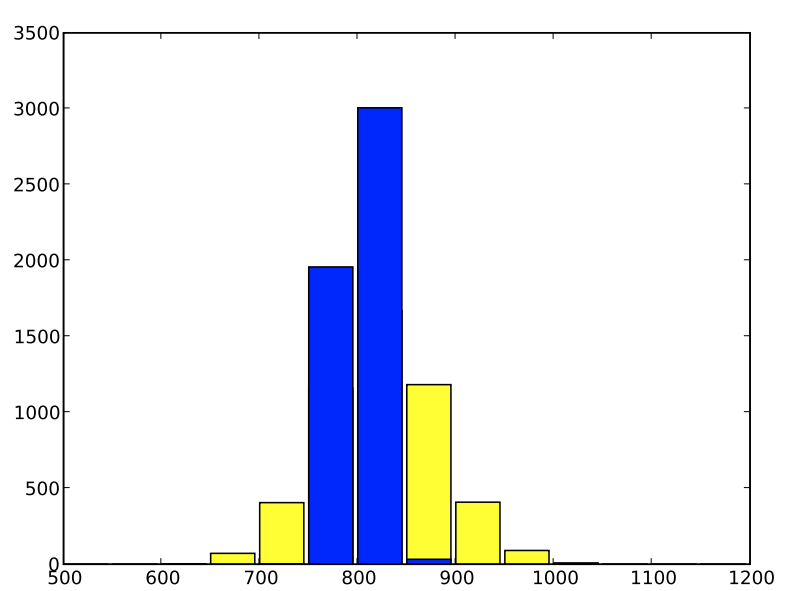

Fig. 13. Distribution of velocity dispersions for 5000 realizations of simulated populations of 1000 (dark) and 100 stars (light), using actual parameters for observational errors.

color-magnitude diagram into some sub-sample with giants, and another with turnoff and upper main-sequence stars. These two subsamples closely correspond to our division between giants and dwarfs. For dwarfs, they find dispersion velocities that are on average almost 1.3 times higher than for giants (their Table 3 ).

We also note that the tangential and radial velocity dispersions derived by Loktin et al. (2005) are substantially larger than our radial-velocity dispersions: 0.84 and $0.77 \mathrm{~km} \mathrm{~s}^{-1}$ for the giants; 1.01 and $1.04 \mathrm{~km} \mathrm{~s}^{-1}$ for dwarfs ("radial" in their work refers to polar coordinates in the sky plane). Loktin et al. (2005) adopt a distance to the cluster of 904 parsecs. Using our preferred distance of $831 \mathrm{pc}$, the radial and tangential dispersions become $(0.77,0.71)$ and $(0.93,0.96) \mathrm{km} \mathrm{s}^{-1}$, respectively for giants and dwarfs, fully compatible with the observed radial velocity dispersions of 0.68 (giants) and $0.9 \mathrm{~km} \mathrm{~s}^{-1}$ (dwarfs). This result indirectly confirms the shorter distance scale for this cluster that emerges from recent works (cf. Pasquini et al. 2008).

It is remarkable that in both our data and those by Mathieu et al. (1986), the ratio of the radial velocity dispersions of mainsequence to evolved stars is 1.3 , the same as found by Zhao et al. (1996) and Loktin et al. (2005) for the dispersion between fainter and brighter stars. Zhao et al. (1996) interpret these results claiming that the dynamical energy of the more massive stars has been passed in the time evolution of the cluster to the less massive objects, which are now escaping the cluster.

While a full dynamical analysis of the cluster is beyond the scope of this work, the possibility of joining precise radial velocity and proper motion data seems attractive for detailed future investigations.

M67 has become the most well-studied open cluster in terms of the number of stars observed and the precision of the measurements. Substantially larger samples of radial velocity measurements have been gathered in globular clusters (see e.g. Sommariva et al. 2009), but they are dynamically much more complex objects with larger velocity dispersions.

\section{Conclusions}

We have carried out a search to find expected differences in the gravitational redshifts of the spectra of dwarf and giant stars in the M67 open cluster, whose stars should share the same systemic velocity, and where the average radial motion of different types of stars should thus produce the same Doppler shift. However, despite an adequate measurement precision, the expected signature has not been seen. Investigations of the reasons for absence of signal - in particular following high-resolution spectral synthesis from 3D hydrodynamic model atmospheres - indicate that likely reasons are different amounts of convective blushifts in different types of stars. An enhanced convective blueshift in, particularly, those stronger spectral lines in dwarf stars that carry a larger weight in the cross-correlation techniques used for radial-velocity measurement, appear adequate to cancel the expected gravitational redshift.

This study thus also illustrates the limitations of crosscorrelation techniques for high-accuracy lineshift measurements. To understand the origin of wavelength shifts that are only a small fraction of any spectral line width, a crosscorrelation performed over an extended spectral range may not be adequate, but rather one needs to examine (groups of) individually selected line profiles measured with sufficient spectral fidelity to identify also their intrinsic asymmetries and the amount of rotational broadening. These can then be compared to synthetic spectral lines from 3D atmospheres complete with convective blueshifts and gravitational redshifts. Not many current spectrometers have adequate performance to achieve these tasks, but work is in progress towards realizing such high-fidelity spectroscopy with an accurate wavelength calibration at the largest telescopes (Dravins 2010; Pepe et al. 2010; Pasquini et al. 2010). With these instruments, it will be possible to extend the present study to less well explored stars, for instance in the metal-poor and metal-rich regimes.

Acknowledgements. The 3D model of the giant star was computed at the CINECA supercomputing centre, which granted time through the INAFCINECA agreement 2006, 2007. L.P. thanks Paul Bristow for a critical reading of the manuscript. D.D. acknowledges support by the Swedish Research Council and The Royal Physiographic Society in Lund. The suggestions by the referee, Dr. D. Pourbaix, helped to improve the manuscript.

\section{References}

Adams, W. S. 1925, Observatory, 48, 337

Allende Prieto, C., Asplund, M., García López, R. J., \& Lambert, D. L. 2002a, ApJ, 567, 544

Allende Prieto, C., Lambert, D. L., Tull, R. G., \& MacQueen, P. J. 2002b, ApJ, 566, L93

Alonso, A., Arribas, S., \& Martínez-Roger, C. 1996, A\&A, 313, 873

Alonso, A., Arribas, S., \& Martínez-Roger, C. 1999, A\&AS, 140, 261; Erratum: 2001, A\&A, 376, 1039

An, D., Terndrup, D. M., Pinsonneault, M. H., et al. 2007, ApJ, 655, 233

Asplund, M. 2005, ARA\&A, 43, 481

Asplund, M., Nordlund, Å., Trampedach, R., Allende Prieto, C., \& Stein, R. F. 2000, A\&A, 359, 729

Bagnulo, S., Jehin, E., Ledoux, C., et al. 2003, ESO Messenger, 114, 10

Baranne, A., Mayor, M., \& Poncet, J. L. 1979, Vist. Astron., 23, 279

Baranne, A., Queloz, D., Mayor, M., et al. 1996, A\&AS, 119, 373

Beckers, J. M. 1977, ApJ, 213, 900

Bouchy, F., Pepe, F., \& Queloz, D. 2001, A\&A, 374, 733

Conti, P. S., Leep, E. M., \& Lorre, J. J. 1977, ApJ, 214, 759

Dravins, D. 1982, ARA\&A, 20, 61

Dravins. D. 2005, in Modelling of Stellar Atmospheres, ed. N. Piskunov, W. W. Weiss, \& D. F. Gray, IAU Symp., 210, E4

Dravins, D. 2008, A\&A, 492, 199

Dravins, D. 2010, Astron. Nachr., 331, 535

Dravins, D., \& Nordlund, Å. 1990, A\&A, 228, 203

Dravins, D., Lindegren, L., Ludwig, H.-G., \& Madsen, S. 2005, in 13th Cambridge Workshop on Cool Stars, Stellar Systems and the Sun, ed. F. Favata, G. Hussain, \& B. Battrick, ESA SP-560, I, 113

Girard, T. M., Grundy, W. M., Lopez, C. E., \& van Altena, W. F. 1989, AJ, 98, 227

Girardi, L., Bressan, A., Bertelli, G., \& Chiosi, C. 2000, A\&AS, 141, 371

Greenstein, J. L., Oke, J. B., \& Shipman, H. 1985, QJRAS, 26, 279 
Griffin, R. F. 1982, J. Astrophys. Astr., 3, 383

Hentschel, K. 1994, Archive for History of Exact Sciences, 47, 143

Hetherington, N. S. 1980, QJRAS, 21, 246

Kaufer, A., Stahl, O., Tubbesing, S., et al. 1999, ESO Messenger, 95, 8

Kurucz, R. L., Furenlid, I., Brault, J., \& Testerman, L. 1984, Solar Flux Atlas from 296 to $1300 \mathrm{~nm}$, Sunspot, New Mexico: National Solar Observatory

Latham, D. W., Mathieu, R. D., Milone, A. A. E., \& Davis, R. J. 1992, IAU Coll. 135: Complementary Approaches to Double and Multiple Star Research, ed. H. A. McAlister, \& W. I. Hartkopf, ASPC, 32, 155

Lindegren, L., \& Dravins, D. 2003, A\&A, 401, 1185

Loktin, A. V. 2005, Astron. Rep. 49, 693 [AZh, 82, 779]

Ludwig, H.-G., Caffau, E., Steffen, M., et al. 2009, Mem. Soc. Astron. Ital., 80, 711

Madsen, S., Dravins, D., \& Lindegren, L. 2002, A\&A, 381, 446

Mathieu, R. D., Latham, D. W., Griffin, R. F., \& Gunn, J. E. 1986, AJ, 92, 1100

Melo, C. H. F., Pasquini, L., \& De Medeiros, J. R. 2001, A\&A, 375, 851 (Paper I)

Montgomery, K. A., Marschall, L. A., \& Janes, K. A. 1993, AJ, 106, 181

Nagendra, K. N., Bonifacio, P., \& Ludwig, H.-G. 2009, 3D Views on Cool Stellar Atmospheres: Theory meets observation, Mem. Soc. Astron. Ital., 80, 601

Nordström, B., Andersen, J., \& Andersen, M. I. 1997, A\&A, 322, 460

Pace, G., Pasquini, L., \& François, P. 2008, A\&A, 489, 403

Pasquini, L., Randich, S., Zoccali, M., et al. 2004, A\&A, 424, 951

Pasquini, L., Biazzo, K., Bonifacio, P., Randich, S., \& Bedin, L. R. 2008, A\&A, 489,677
Pasquini, L., Cristiani, S., García López, R., et al. 2010, Proc. SPIE, 7735, $77352 \mathrm{~F}$

Pepe, F. A., Cristiani, S., Rebolo Lopez, R., et al. 2010, Proc. SPIE, 7735, $77350 \mathrm{~F}$

Platais, I., Melo, C., Mermilliod, J.-C., et al. 2007, A\&A, 461, 509

Pourbaix, D., Nidever, D., McCarthy, C., et al. 2002, A\&A, 386, 280

Pryor, C., \& Meylan, G. 1993, Structure and Dynamics of Globular Clusters, ed. S. G. Djorgovski, \& G. Meylan, ASPC, 50, 357

Ramírez, I., Allende Prieto, C., \& Lambert, D. L. 2008, A\&A, 492, 841

Randich, S., Sestito, P., Primas, F., Pallavicini, R., \& Pasquini, L. 2006, A\&A, 450,557

Sanders, W. L. 1977, A\&AS, 27, 89

Sandquist, E. L. 2004, MNRAS, 347, 101

Sommariva, V., Piotto, G., Rejkuba, M., et al. 2009 A\&A, 493, 947

Setiawan, J., Pasquini, L., da Silva, L., et al. 2004, A\&A, 421, 241

Sousa, S. G., Santos, N. C., Mayor, M., et al. 2008, A\&A, 487, 373

Tautvaišienė, G., Edvardsson, B., Tuominen, I., \& Ilyin, I. 2000, A\&A, 360, 499

Taylor, B. J. 2007, AJ, 133, 370

Torres, C. A. O., Quast, G. R., da Silva, L., et al. 2006, A\&A, 460, 695

Trumpler, R. J. 1935, PASP, 47, 249

WEBDA 2009, http: //www . univie.ac .at/webda/

von Hippel, T. 1996, ApJ, 458, L37

Zhao, J. L., Tian, K. P., \& Su, C. G. 1996, Ap\&SS, 235, 93 
Table 1. Observed targets in M 67.

\begin{tabular}{|c|c|c|c|c|c|}
\hline Object & $B-V$ & $\bar{V}$ & RV & $V \sin i$ & Source \\
\hline S 986 & 0.554 & 12.73 & 34.08 & 4.5 & Paper I \\
\hline S 988 & 0.574 & 13.18 & 34.28 & 6.6 & Paper I \\
\hline S 989 & 1.057 & 11.45 & 34.66 & $<1.3$ & Paper I \\
\hline S 994 & 0.581 & 13.18 & 34.36 & 6.3 & Paper I \\
\hline S 995 & 0.559 & 12.76 & 34.54 & 4.9 & Paper I \\
\hline S 998 & 0.567 & 13.06 & 34.64 & 6.3 & Paper I \\
\hline S 1001 & 0.990 & 12.40 & 33.38 & $<1.3$ & Paper I \\
\hline S 1010 & 1.110 & 10.48 & 33.95 & $<1.3$ & Paper I \\
\hline S 1011 & 0.627 & 13.82 & 27.23 & 3.9 & Paper I \\
\hline S 1016 & 1.260 & 10.30 & 34.80 & $<1.3$ & Paper I \\
\hline S 1034 & 0.608 & 12.65 & 34.47 & 4.6 & Paper I \\
\hline S 1055 & 0.586 & 13.79 & 34.32 & 4.3 & Paper I \\
\hline S 1064 & 0.660 & 14.04 & 20.40 & 4.6 & Paper I \\
\hline S 1074 & 1.120 & 10.59 & 33.94 & $<1.3$ & Paper I \\
\hline S 1075 & 0.598 & 13.84 & 33.61 & 4.6 & Paper I \\
\hline S 1221 & 1.130 & 10.76 & 30.37 & $<1.3$ & Paper I \\
\hline S 1231 & 0.917 & 12.93 & 32.87 & $<1.3$ & Paper I \\
\hline S 1239 & 0.758 & 12.75 & 32.55 & 2.8 & Paper I \\
\hline S 1250 & 1.360 & 9.69 & 36.05 & $<1.3$ & Paper I \\
\hline S 1256 & 0.660 & 13.67 & 33.61 & 3.4 & Paper I \\
\hline S 1279 & 1.120 & 10.55 & 33.34 & $<1.3$ & Paper I \\
\hline S 1288 & 1.070 & 11.33 & 33.17 & $<1.3$ & Paper I \\
\hline S 1293 & 1.007 & 12.09 & 33.59 & 1.9 & Paper I \\
\hline S 1305 & 0.993 & 12.23 & 33.22 & 3.0 & Paper I \\
\hline S 1314 & 0.635 & 13.70 & 27.10 & 4.1 & Paper I \\
\hline S 1316 & 1.100 & 10.58 & 32.81 & $<1.3$ & Paper I \\
\hline S 1479 & 1.100 & 10.55 & 34.40 & $<1.3$ & Paper I \\
\hline S 2205 & 0.566 & 13.14 & 31.25 & 7.6 & Paper I \\
\hline S 598 & 0.600 & 12.63 & 33.64 & 4.1 & Run II \\
\hline S 602 & 0.550 & 12.95 & 34.05 & 4.2 & Run II \\
\hline S 610 & 0.540 & 12.89 & 33.79 & 7.4 & Run II \\
\hline S 615 & 0.550 & 12.82 & 34.59 & 6.5 & Run II \\
\hline S 656 & 0.570 & 13.15 & 33.82 & 4.2 & Run II \\
\hline S 657 & 0.610 & 12.60 & 33.18 & 4.0 & Run II \\
\hline S 711 & 0.580 & 13.37 & 21.36 & 4.6 & Run II \\
\hline S 723 & 0.580 & 13.35 & 34.28 & 7.0 & Run II \\
\hline S 731 & 0.534 & 13.06 & 33.43 & 4.6 & Run II \\
\hline S 736 & 0.554 & 13.37 & 33.75 & 4.2 & Run II \\
\hline S 745 & 0.540 & 13.17 & 48.10 & 5.7 & Run II \\
\hline S 760 & 0.575 & 13.29 & 36.52 & 6.7 & Run II \\
\hline S 773 & 0.589 & 13.31 & -16.66 & 11.3 & Run II \\
\hline S 809 & 0.510 & 13.47 & 33.45 & 4.8 & Run II \\
\hline S 815 & 0.550 & 12.77 & 34.28 & 6.4 & Run II \\
\hline S 816 & 0.580 & 13.22 & 25.33 & 4.9 & Run II \\
\hline S 827 & 0.590 & 13.10 & 35.16 & 6.3 & Run II \\
\hline S 926 & 0.550 & 12.71 & 33.69 & 5.2 & Run II \\
\hline S 927 & 0.540 & 13.31 & 34.87 & 6.3 & Run II \\
\hline S 967 & 0.578 & 13.39 & 34.22 & 4.7 & Run II \\
\hline S 1003 & 0.572 & 12.80 & 33.02 & 7.3 & Run II \\
\hline S 1011 & 0.627 & 13.82 & 29.10 & 4.4 & Run II \\
\hline S 1016 & 1.260 & 10.30 & 34.72 & $<1.3$ & Run II \\
\hline S 1017 & 0.563 & 13.37 & 32.32 & 6.8 & Run II \\
\hline S 1030 & 0.566 & 13.23 & 34.65 & 6.9 & Run II \\
\hline S 1032 & 0.573 & 13.48 & 34.17 & 5.7 & Run II \\
\hline S 1034 & 0.608 & 12.65 & 34.42 & 4.7 & Run II \\
\hline S 1046 & 0.571 & 13.51 & 30.95 & 5.3 & Run II \\
\hline S 1049 & 0.565 & 12.82 & 32.45 & 7.4 & Run II \\
\hline S 1055 & 0.586 & 13.79 & 35.31 & 3.8 & Run II \\
\hline S 1062 & 0.594 & 13.29 & 32.22 & 7.0 & Run II \\
\hline S 1071 & 0.589 & 12.81 & 32.92 & 5.1 & Run II \\
\hline S 1075 & 0.598 & 13.84 & 33.74 & 4.5 & Run II \\
\hline S 1076 & 0.567 & 12.83 & 34.83 & 4.2 & Run II \\
\hline S 1181 & 0.570 & 13.19 & 33.43 & 6.8 & Run II \\
\hline S 1186 & 0.580 & 12.75 & 33.52 & 5.2 & Run II \\
\hline S 1189 & 0.573 & 13.29 & 34.32 & 5.7 & Run II \\
\hline S 1197 & 0.560 & 13.18 & 34.41 & 6.8 & Run II \\
\hline S 1207 & 0.570 & 13.24 & 33.03 & 6.4 & Run II \\
\hline
\end{tabular}


Table 1. continued.

\begin{tabular}{|c|c|c|c|c|c|}
\hline Object & $B-V$ & $\bar{V}$ & RV & $\overline{V \sin i}$ & Source \\
\hline S 1219 & 0.597 & 13.15 & 32.57 & 4.8 & Run II \\
\hline S 1220 & 0.572 & 13.37 & 33.34 & 6.9 & Run II \\
\hline S 1226 & 0.575 & 13.34 & 35.06 & 4.2 & Run II \\
\hline S 1230 & 0.559 & 13.08 & 32.77 & 5.6 & Run II \\
\hline S 1240 & 0.572 & 13.38 & 34.22 & 5.5 & Run II \\
\hline S 1252 & 0.643 & 14.07 & 31.66 & 3.8 & Run II \\
\hline S 1260 & 0.625 & 14.19 & 34.79 & 3.7 & Run II \\
\hline S 1268 & 0.581 & 12.65 & 32.85 & 4.0 & Run II \\
\hline S 1270 & 0.577 & 12.73 & 31.72 & 4.9 & Run II \\
\hline S 1271 & 0.534 & 12.91 & 34.10 & 7.9 & Run II \\
\hline S 1302 & 0.564 & 13.08 & 32.90 & 4.4 & Run II \\
\hline S 1310 & 0.580 & 12.76 & 32.27 & 7.4 & Run II \\
\hline S 1321 & 0.560 & 13.54 & 34.37 & 4.3 & Run II \\
\hline S 1337 & 0.590 & 13.00 & 31.65 & 4.6 & Run II \\
\hline S 1429 & 0.560 & 12.75 & 33.25 & 4.6 & Run II \\
\hline S 1441 & 0.572 & 13.15 & 33.67 & 6.5 & Run II \\
\hline S 1456 & 0.575 & 12.71 & 32.35 & 4.8 & Run II \\
\hline S 1458 & 0.577 & 13.36 & 35.22 & 6.2 & Run II \\
\hline S 1459 & 0.530 & 13.47 & 34.27 & 6.2 & Run II \\
\hline S 1464 & 0.570 & 13.28 & 34.48 & 3.7 & Run II \\
\hline S 1471 & 0.595 & 12.83 & 33.05 & 5.1 & Run II \\
\hline S 1485 & 0.560 & 12.78 & 34.95 & 8.6 & Run II \\
\hline S 1487 & 0.640 & 12.63 & 34.29 & 3.7 & Run II \\
\hline S 1583 & 0.560 & 13.21 & 33.12 & 6.9 & Run II \\
\hline S 1589 & 0.540 & 12.76 & 32.82 & 5.0 & Run II \\
\hline S 1607 & 0.560 & 12.62 & 33.42 & 4.2 & Run II \\
\hline S 2207 & 0.617 & 12.65 & 34.66 & 4.9 & Run II \\
\hline S 2219 & 0.557 & 13.17 & 32.37 & 11.5 & Run II \\
\hline S 2220 & 0.578 & 13.17 & 33.54 & 4.5 & Run II \\
\hline MMJ5313 & 0.733 & 13.36 & -3.83 & 23.7 & Run III \\
\hline MMJ5336 & 1.107 & 12.96 & -7.54 & $<1.3$ & Run III \\
\hline MMJ5877 & 1.006 & 12.11 & 37.57 & 1.3 & Run III \\
\hline MMJ5927 & 0.822 & 12.78 & 34.83 & 3.1 & Run III \\
\hline MMJ6034 & 1.000 & 12.91 & 35.65 & 3.1 & Run III \\
\hline MMJ6470 & 1.360 & 9.84 & 33.16 & $<1.3$ & Run III \\
\hline MMJ6471 & 1.590 & 8.86 & 32.77 & $<1.3$ & Run III \\
\hline MMJ6472 & 1.100 & 9.98 & 44.84 & 2.3 & Run III \\
\hline MMJ6474 & 1.230 & 10.52 & 9.32 & 0.6 & Run III \\
\hline MMJ6489 & 1.080 & 11.20 & 33.40 & 1.3 & Run III \\
\hline MMJ6495 & 1.480 & 9.37 & 34.03 & $<1.3$ & Run III \\
\hline MMJ6499 & 1.360 & 9.69 & 34.31 & $<1.3$ & Run III \\
\hline MMJ6510 & 0.110 & 10.70 & -24.57 & 1.5 & Run III \\
\hline MMJ6513 & 1.230 & 10.02 & 67.59 & 0.3 & Run III \\
\hline MMJ6514 & 1.670 & 8.74 & 34.09 & 0.9 & Run III \\
\hline MMJ6515 & 1.300 & 10.12 & 33.93 & $<1.3$ & Run III \\
\hline S 219 & 0.960 & 16.06 & 34.11 & 6.9 & Run III \\
\hline S 266 & 0.770 & 13.87 & 33.78 & 4.4 & Run III \\
\hline S 288 & 0.580 & 15.48 & 36.97 & 5.2 & Run III \\
\hline S 291 & - & - & 32.52 & $<1.3$ & Run III \\
\hline S 606 & 0.950 & 12.77 & 34.04 & 1.4 & Run III \\
\hline S 613 & 0.900 & 15.50 & 33.78 & 4.1 & Run III \\
\hline S 694 & 0.970 & 12.83 & 36.22 & 1.6 & Run III \\
\hline S 721 & 1.100 & 11.24 & 35.34 & 0.8 & Run III \\
\hline S 774 & 0.870 & 12.95 & 33.57 & 2.5 & Run III \\
\hline S 774 & 0.860 & 12.93 & 33.57 & 2.5 & Run III \\
\hline S 794 & 0.941 & 12.86 & 33.76 & 2.1 & Run III \\
\hline S 806 & 0.813 & 12.78 & 33.57 & 3.8 & Run III \\
\hline S 978 & 1.370 & 9.72 & 34.53 & $<1.3$ & Run III \\
\hline S 989 & 1.057 & 11.45 & 34.72 & 1.5 & Run III \\
\hline S 1001 & 0.990 & 12.40 & 33.32 & $<1.3$ & Run III \\
\hline S 1010 & 1.110 & 10.48 & 33.59 & 2.1 & Run III \\
\hline S 1016 & 1.260 & 10.30 & 34.00 & $<1.3$ & Run III \\
\hline S 1034 & 0.608 & 12.65 & 34.46 & 5.6 & Run III \\
\hline S 1051 & 0.605 & 13.93 & 32.98 & 4.8 & Run III \\
\hline S 1055 & 0.586 & 13.79 & 35.10 & 4.9 & Run III \\
\hline S 1056 & 0.854 & 13.02 & 33.75 & 2.8 & Run III \\
\hline S 1074 & 1.120 & 10.59 & 34.04 & 2.0 & Run III \\
\hline
\end{tabular}


Table 1. continued.

\begin{tabular}{|c|c|c|c|c|c|}
\hline Object & $B-V$ & $\bar{V}$ & RV & $V \sin i$ & Source \\
\hline S 1075 & 0.598 & 13.84 & 33.27 & 5.9 & Run III \\
\hline S 1084 & 1.100 & 10.48 & 33.87 & 2.3 & Run III \\
\hline S 1090 & 0.810 & 15.31 & 35.10 & 5.1 & Run III \\
\hline S 1103 & 0.940 & 12.40 & 34.34 & 2.1 & Run III \\
\hline S 1231 & 0.917 & 12.93 & 33.12 & 2.4 & Run III \\
\hline S 1245 & 0.919 & 12.93 & 33.09 & 2.0 & Run III \\
\hline S 1254 & 1.050 & 11.52 & 33.02 & 1.6 & Run III \\
\hline S 1277 & 1.050 & 11.63 & 34.32 & 1.6 & Run III \\
\hline S 1279 & 1.120 & 10.55 & 33.48 & 2.2 & Run III \\
\hline S 1288 & 1.070 & 11.33 & 33.54 & 1.2 & Run III \\
\hline S 1293 & 1.007 & 12.09 & 34.16 & 1.7 & Run III \\
\hline S 1303 & 0.601 & 13.71 & 33.13 & 5.0 & Run III \\
\hline S 1305 & 0.993 & 12.23 & 34.07 & 2.0 & Run III \\
\hline S 1315 & 0.980 & 15.91 & 33.84 & 4.3 & Run III \\
\hline S 1316 & 1.100 & 10.58 & 32.94 & 2.1 & Run III \\
\hline S 1319 & 0.920 & 12.90 & 34.17 & 2.2 & Run III \\
\hline S 1402 & 1.150 & 10.93 & 33.82 & 0.9 & Run III \\
\hline S 1438 & 0.817 & 12.89 & 32.81 & 3.1 & Run III \\
\hline S 1463 & 1.010 & 12.94 & 33.97 & 1.1 & Run III \\
\hline S 1479 & 1.100 & 10.55 & 34.33 & 2.1 & Run III \\
\hline S 1524 & 0.810 & 13.00 & 33.25 & 3.2 & Run III \\
\hline S 1557 & 1.260 & 10.10 & 33.86 & $<1.3$ & Run III \\
\hline S 1585 & 0.940 & 12.50 & 33.31 & 1.7 & Run III \\
\hline S 1592 & 1.120 & 10.47 & 33.64 & 2.3 & Run III \\
\hline S 1679 & 0.310 & 12.38 & 13.19 & 26.2 & Run III \\
\hline S 1758 & 1.640 & 11.20 & 32.04 & 3.1 & Run III \\
\hline S 1808 & 0.580 & 12.60 & 31.61 & 4.9 & Run III \\
\hline S 1852 & 0.840 & 16.15 & 33.00 & 3.9 & Run III \\
\hline S 2015 & 0.610 & 12.56 & 33.59 & 5.4 & Run III \\
\hline
\end{tabular}

Notes. Stars $B-V$ colors, apparent $V$ magnitudes, and spectroscopic data. For object identifications, "S" are from Sanders (1977); "MMJ" from Montgomery et al. (1993). Radial velocities (RV) and projected rotational velocities $V \sin i$ are in $\mathrm{km} \mathrm{s}^{-1}$; the data sources are either Paper I (Melo et al. 2001) or the observing runs decribed in Sect. 3. 